\title{
Unexpected Nectriaceae species diversity in cheese, description of Bisifusarium allantoides sp. nov., Bisifusarium penicilloides sp. nov., Longinectria gen. nov. lagenoides sp. nov. and Longinectria verticilliforme sp. nov.
}

\author{
Savary $\mathrm{O}^{1}$, Coton $\mathrm{M}^{1}$, Frisvad $\mathrm{JC}^{2}$, Nodet $\mathbf{P}^{1}$, Ropars $\mathrm{J}^{3}$, Coton $\mathbf{E}^{1}$ and \\ Jany J-L $\mathbf{L}^{1 \#}$
}

${ }^{1}$ Univ Brest, Laboratoire Universitaire de Biodiversité et Écologie Microbienne, F-29280 Plouzané, France

${ }^{2}$ Department of Biotechnology and Biomedecine, Technical University of Denmark, DK-2800 Kgs. Lyngby, Denmark

${ }^{3}$ Ecologie Systématique et Evolution, Université Paris-Saclay, CNRS, AgroParisTech, 91400 Orsay, France

Savary O, Coton M, Frisvad JC, Nodet P, Ropars J, Coton E, Jany J-L 2021 - Unexpected Nectriaceae species diversity in cheese, description of Bisifusarium allantoides sp. nov., Bisifusarium penicilloides sp. nov., Longinectria gen. nov. lagenoides sp. nov. and Longinectria verticilliforme sp. nov. Mycosphere 12(1), 1077-1100, Doi 10.5943/mycosphere/12/1/13

\begin{abstract}
Investigation of various cheese rinds for Bisifusarium domesticum revealed a completely unexpected diversity of Fusarium-like fungi. Elongation factor 1-alpha gene sequence data, classically used for Fusarium spp. identification, suggested that some isolates corresponded to so far undescribed Nectriaceae species. In this context, a multi-gene phylogenetic analysis, based on ten loci, namely the large subunit of the ATP citrate lyase (acll), alpha-actin (act), calmodulin ( $c m d A)$, histone H3 (his3), the internal transcribed spacer region and intervening 5.8S nrRNA (ITS), 28S large subunit ( $L S U)$, RNA polymerase II largest subunit ( $r p b 1)$, RNA polymerase II second largest subunit $(r p b 2)$, translation elongation factor 1-alpha (tefl) and $\beta$-tubulin (tub2), was therefore performed to determine the phylogenetic position of ten cheese and one environmental isolates. Phylogenetic reconstructions were then done using a Nectriaceae sequence dataset. Additionally, morphological observations as well as metabolite profiling were performed. Results clearly revealed the existence of four novel cheese-associated species, namely Bisifusarium allantoides, Bisifusarium penicilloides, Longinectria lagenoides, Longinectria verticilliforme, the two latter belonging to a novel genus (Longinectria gen. nov.).
\end{abstract}

Keywords - Fungi - Fusarium-like - Phylogeny - Taxonomy

\section{Introduction}

Cheese making dates back at least 8000 years (Fox \& Guinee 2020) and in some cheeses, fungi play a major role during cheese ripening by contributing to their organoleptic richness and diversity (Irlinger et al. 2015). The most well-known examples worldwide are probably moldy rind soft cheeses (e.g. Brie and Camembert) produced using Penicillium camemberti and Geotrichum candidum, and blue-veined cheeses (e.g. Roquefort, Gorgonzola, Stilton) produced with Penicillium roqueforti (Cheeseman et al. 2014, Gillot et al. 2017, Dumas et al. 2020). Beyond these emblematic examples, other moulds contribute to cheese making (e.g. Mucor spp., Scopulariopsis spp. or 
Bisifusarium domesticum) (Hermet et al. 2012, Morin-Sardin et al. 2016, Dupont et al. 2017, Lebreton et al. 2020). During the last decades, many studies have revealed that cheeses harbour substantial fungal diversity (Ropars et al. 2012, Wolfe et al. 2014, Hermet et al. 2014, Dugat-Bony et al. 2016). These fungi may be either indigenous, originating from the ripening rooms or cheese environment, or voluntarily inoculated as adjunct cultures. At least part of the main species associated with cheese making are thought to have undergone a domestication process (i.e. adaptive divergence under human pressure at a human time scale), as observed for the emblematic $P$. camemberti and $P$. roqueforti species (Ropars et al. 2015, 2016, 2017, 2020, Bodinaku et al. 2019, Dumas et al. 2020), or have been described as well adapted to the cheese substrate, such as Mucor spp. found in Tomme (Morin-Sardin et al. 2016). Besides the above-mentioned species, which have been extensively studied, common cheese taxa such as Scopulariopsis spp. or B. domesticum have been seldomly investigated (Ropars et al. 2012). The latter species is commonly used by cheesemakers as an adjunct culture for cheese productions (e.g. Saint-Nectaire, Reblochon), and in some cases, to prevent excessive surface stickiness (e.g. Raclette, Gruyère, Appenzeller), a property from which its trivial name "Anticollanti" is derived (Bachmann et al. 2003, 2005). To date, B. domesticum has been solely isolated from cheese and is the only species within the family Nectriaceae associated with this food matrix. It was originally identified as Trichothecium domesticum, then described as Fusarium domesticum by Bachman et al. (Bachmann et al. 2003, 2005). Recently, nomenclatural changes divided Fusarium into seven different genera (Lombard et al. 2015, Sandoval-Denis et al. 2018, 2019). Fusarium domesticum was therefore assigned to the new genus Bisifusarium (and named $B$. domesticum) along with six other taxa previously assigned to the Fusarium dimerum species complex (FDSC) (Schroers et al. 2009, Lombard et al. 2015), namely B. biseptatum (Schroers, Summerbell \& O’Donnell) Lombard \& Crous, 2015, B. delphinoides (Schroers, Summerbell, O'Donnell \& Lampr.) Lombard \& Crous, 2015, B. dimerum (Penz.) Lombard \& Crous 2015, B. domesticum (Fr.) Lombard \& Crous 2015, B. lunatum (Ellis \& Everh.) Lombard \& Crous 2015, B. nectrioides (Wollenw.) Lombard \& Crous 2015 and B. penzigii (Schroers, Summerbell \& O'Donnell) L. Lombard \& Crous, 2015 (Lombard et al. 2015). An eighth species, Bisifusarium tonghuanum B.D. Sun, Y.G. Zhou \& A.J. Chen, 2017, was also recently added to the genus (Sun et al. 2017). Species within the genus Bisifusarium were described as having short macroconidia with a maximum of three septa and short phialides arising as lateral pegs from the hyphae (Gerlach \& Nirenberg 1982, Schroers et al. 2009, Lombard et al. 2015, Sun et al. 2017).

It is noteworthy that the circumscription of the Fusarium genus, and consecutively the definition of Bisifusarium has recently been highly debated (Crous et al. 2021 vs Geiser et al. 2021) and, so far, no consensus nomenclature has been provided.

Accessing a large number of isolates is crucial for studying a species of interest. We therefore investigated different cheeses to create a large working collection of B.domesticum (F. domesticum sensu Geiser et al. 2020) isolates which led to the isolation and identification of various Fusariumlike isolates. Sequencing of the translation elongation factor 1-alpha used for rapid isolate identifications highlighted an unexpected diversity within the Nectriaceae family for four putative novel taxa. In order to clarify the taxonomic position of these four taxa, a multi-gene phylogenetic analysis using the ten gene set described by Lombard et al. (2015) was performed. To complete this description, morphological characters and secondary metabolite profiles were determined.

\section{Material \& Methods}

\section{Fungal isolations from cheeses}

In total, fifty-six cheeses were analysed. On the one hand, forty-six different cheeses (from France, Switzerland, Italy, Spain and Canada), including artisanal and industrial cheeses, were obtained for fungal isolations. For each cheese sample, $10 \mathrm{~g}$ of rind were removed using a sterile scalpel, transferred into $90 \mathrm{~mL}$ of Tween $80(0.015 \% \mathrm{v} / \mathrm{v})$ in a sterile stomacher bag equipped with a filter membrane and mixed for 180 seconds using a Stomacher homogenizer (AES, France). Serial dilutions of the homogenate were spread on dichloran glycerol chloramphenicol selective agar 
(DG18, Millipore, Merck, Germany) and malt yeast extract agar (M2Lev) supplemented with antibiotics $(20 \mathrm{~g} / \mathrm{L}$ malt extract, $3 \mathrm{~g} / \mathrm{L}$ yeast extract $15 \mathrm{~g} / \mathrm{L}$ agar, $100 \mathrm{mg} / \mathrm{L}$ penicillin and $100 \mathrm{mg} / \mathrm{L}$ streptomycin). Agar plates were incubated at $25^{\circ} \mathrm{C}$ for 7 days. Fungal colonies harbouring typical macroscopic and/or microscopic characters of B. domesticum or Fusarium-like fungi were further isolated and purified on M2Lev medium. On the other hand, analysis of ten different cheeses by international colleagues allowed us to increase the number of Fusarium-like isolates. All isolates were conserved at $-80^{\circ} \mathrm{C}$ in $10 \%$ glycerol (v/v).

\section{DNA extraction, PCR amplification and sequencing}

DNA extractions were performed on seven to ten-day monospore cultures grown on M2Lev medium using the FastDNA SPIN kit (MP Biomedicals, Fisher Scientific) according to manufacturer recommendations. Each DNA extract was diluted to $40 \mathrm{ng} / \mu \mathrm{L}$ and stored at $-20^{\circ} \mathrm{C}$ until PCR amplification.

Firstly, sequencing of the translation elongation factor 1-alpha (tefl) was used for species identification. Generated sequence data was compared to the GenBank database using the Basic Local Alignment Search Tool (BLAST). Then, a subset of thirteen representative isolates, including eleven unidentified ones, was selected and used for the phylogenetic study (Table 1).

Secondly, their phylogenetic position was assessed using the ten loci and same primers as those described by Lombard et al. (2015) which was the only set available at the beginning of this study. Pending adoption of a consensus taxonomy, we named taxa according to the nomenclature proposed by the same authors. The ten loci corresponded to the large subunit of the ATP citrate lyase (acll), alpha-actin (act), calmodulin ( $c m d A$ ), histone $\mathrm{H} 3$ (his3), the internal transcribed spacer region and intervening 5.8S nrRNA (ITS), 28S large subunit ( $L S U)$, RNA polymerase II largest subunit (rpbl), RNA polymerase II second largest subunit (rpb2), translation elongation factor 1-alpha (tefl) and $\beta$ tubulin (tub2) genes. PCR mixtures (Table 2) using the different primers are detailed in Table S1. Specific PCR amplifications were checked after migration of resulting amplicons on a $1 \%(\mathrm{w} / \mathrm{v})$ agarose gel in $1 \mathrm{X}$ Tris-acetate-EDTA buffer (molecular biology grade, Promega). Sequencing was performed using the different forward and reverse primers by Eurofins Genomics platform (Ebersberg, Germany). Contig assembly was done using Geneious prime v2020.0.3. The sequences generated and used in the phylogenetic analysis were deposited in GenBank under accession numbers detailed in Table 1.

\section{Dataset preparation}

Since a preliminary phylogenetic reconstruction performed using tefl sequences nested all the isolates of interest within the IX to XII clades described in Lombard et al. (2015) (data not shown), for each of the ten analyzed loci, a first dataset, consisting of the sequences of the isolates of interest from our collection as well as 57 sequences corresponding to unique representative strains of each taxon from those four clades, was established (Table S2). An additional reduced dataset that included only ITS, LSU, rpb1, rpb2, tef1 and tub2 genes sequences from the first dataset identified as pertaining to Bisifusarium, based on the phylogenetic reconstruction obtained with the full dataset in the present study, was used to confirm that the four loci missing for $B$. tonghuanum had no impact on the positioning of several isolates (despite our best efforts, additional sequence data could not be obtained due to the unavailability of any B. tonghuanum strain).

\section{Alignment and phylogenetic reconstruction}

Sequences of the ten loci: acl1, act, cmdA, his3, ITS, LSU, rpb1, rpb2, tef1 and tub2 were aligned independently using MAAFT v7.450 (Kuraku et al. 2013, Katoh et al. 2019) with the Auto algorithm as implemented in Geneious prime v2020.0.3. Introns and exons were separated in each dataset. Alignment of the exonic region was ascertained by checking the deduced translated aminoacyl alignment and alignment of the intronic regions was edited using Gblocks b0.91b 
(http://molevol.cmima.csic.es/castresana/Gblocks_server.html) with all the options enabled to allow less stringent selection, discard highly ambiguous regions and ensure reproducibility. Since phylogenetic incongruence between the different genes was not detected $(P=0.001)$ by performing an Incongruence Length Difference (ILD) test on 1000 replicates as implemented in PAUP 4.0a166 (Swofford 2003), the whole dataset was used after concatenation of the ten individual alignments.

Table 1 Information for strains sequenced for the phylogenetic analyses

\begin{tabular}{|c|c|c|c|c|c|c|c|c|c|c|c|c|c|c|}
\hline \multirow[t]{2}{*}{ Species } & \multirow[t]{2}{*}{ Isolate } & \multirow[t]{2}{*}{ Substrate } & \multirow{2}{*}{$\begin{array}{l}\text { Collector } \\
\text { and/or } \\
\text { Depositor }\end{array}$} & \multirow[t]{2}{*}{ Locality } & \multicolumn{10}{|c|}{ GenBank Accession No. } \\
\hline & & & & & acl1 & act & cmdA & his3 & ITS & $L S U$ & $r p b 1$ & $r p b 2$ & tef1 & tub2 \\
\hline \multirow{6}{*}{$\begin{array}{l}\text { Bisifusarium } \\
\text { allantoides }\end{array}$} & UBOCC-A- & Cheese & O. Savary & Spain & MW81 & MW811 & MW811 & MW811 & MW654 & MW654 & MW811 & MW811 & MW811 & MW811 \\
\hline & 120035 & (Manchego) & & & 0987 & 002 & 017 & 031 & 536 & 511 & 046 & 060 & 075 & 090 \\
\hline & UBOCC-A- & Soft cheese & F. Deniel & France & MW81 & MW811 & MW811 & MW811 & MW654 & MW654 & MW811 & MW811 & MW811 & MW811 \\
\hline & $120036^{\mathrm{T}}$ & type & & & 0999 & 014 & 029 & 044 & 548 & 523 & 054 & 072 & 087 & 102 \\
\hline & UBOCC-A- & Soft cheese & F. Deniel & France & MW81 & MW811 & MW811 & MW811 & MW654 & MW654 & MW811 & MW811 & MW811 & MW811 \\
\hline & 120037 & type & & & 1000 & 015 & 030 & 040 & 549 & 524 & 055 & 073 & 088 & 103 \\
\hline \multirow{2}{*}{$\begin{array}{l}\text { Bisifusarium } \\
\text { biseptatum }\end{array}$} & CBS & Soil & P. Nelson & South-Africa & MW81 & MW811 & MW811 & MW811 & MW654 & MW654 & MW811 & MW811 & MW811 & MW811 \\
\hline & $110311^{\mathrm{T}}$ & & $\begin{array}{l}\text { \& W. } \\
\text { Marasas }\end{array}$ & & 0998 & 013 & 028 & 039 & 547 & 522 & 053 & 071 & 086 & 101 \\
\hline \multirow{7}{*}{$\begin{array}{l}\text { Bisifusarium } \\
\text { dimerum } \\
\text { Bisifusarium } \\
\text { penicilloides }\end{array}$} & $\begin{array}{l}\text { MNHN RF- } \\
05625^{\mathrm{T}}\end{array}$ & Homo sapiens & $\begin{array}{l}\text { H. Ph. } \\
\text { Endtz }\end{array}$ & Netherlands & $\begin{array}{l}\text { MW81 } \\
0997\end{array}$ & $\begin{array}{l}\text { MW811 } \\
012\end{array}$ & $\begin{array}{l}\text { MW811 } \\
027\end{array}$ & $\begin{array}{l}\text { MW811 } \\
038\end{array}$ & $\begin{array}{l}\text { MW654 } \\
546\end{array}$ & $\begin{array}{l}\text { MW654 } \\
521\end{array}$ & $\begin{array}{l}\text { MW811 } \\
058\end{array}$ & $\begin{array}{l}\text { MW811 } \\
070\end{array}$ & $\begin{array}{l}\text { MW811 } \\
085\end{array}$ & $\begin{array}{l}\text { MW811 } \\
100\end{array}$ \\
\hline & UBOCC-A- & Cheese (Mont & O. Savary & France & MW81 & MW811 & MW811 & MW811 & MW654 & MW654 & MW811 & MW811 & MW811 & MW811 \\
\hline & $120021^{\mathrm{T}}$ & d'Or) & & & 0993 & 008 & 023 & 035 & 542 & 517 & 051 & 066 & & 096 \\
\hline & UBOCC-A- & Cheese (Mont & O. Savary & France & MW81 & MW811 & MW811 & MW811 & MW654 & MW654 & MW811 & MW811 & MW811 & MW811 \\
\hline & 120034 & d'Or) & & & 0992 & 007 & 022 & 034 & 541 & 516 & 050 & 065 & 080 & 095 \\
\hline & VTT-D- & Surface of & Suihko et & Scotland, & MW81 & MW811 & MW811 & MW811 & MW654 & MW654 & MW811 & MW811 & MW811 & MW811 \\
\hline & 041022 & $\begin{array}{l}\text { sandstone } \\
\text { building, } \\
\text { Arbroat Abbey }\end{array}$ & al. & UK & 0986 & 001 & 016 & 041 & 535 & 510 & 045 & 059 & 074 & 089 \\
\hline \multirow{10}{*}{$\begin{array}{l}\text { Fusarium } \\
\text { babinda } \\
\text { Fusarium } \\
\text { solani s.l. } \\
\text { Longinectria } \\
\text { lagenoides }\end{array}$} & UBOCC-A- & Raw cow's & O. Savary & France & MW81 & MW811 & MW811 & MW811 & MW654 & MW654 & - & MW811 & MW811 & MW811 \\
\hline & 120044 & milk cheese & & & 0995 & 010 & 025 & 037 & 544 & 519 & & 068 & & 098 \\
\hline & UBOCC-A- & Raw cow's & O. Savary & France & MW81 & MW811 & MW811 & - & MW654 & MW654 & MW811 & MW811 & MW811 & MW811 \\
\hline & 120045 & milk cheese & & & 0996 & 011 & 026 & & 545 & 520 & 052 & 069 & 084 & 099 \\
\hline & ESE 00140 & Cheese (Tilsit) & J. Ropars & France & MW81 & MW811 & MW811 & MW811 & MW654 & MW654 & MW811 & MW811 & MW811 & MW811 \\
\hline & & & & & 0994 & 009 & 024 & 036 & 543 & 518 & 057 & 067 & 082 & 097 \\
\hline & UBOCC-A- & Cheese & O. Savary & France & MW81 & MW811 & MW811 & MW811 & MW654 & MW654 & MW811 & MW811 & MW811 & MW811 \\
\hline & 120 & $\begin{array}{l}\text { (Raclette } \\
\text { d'alpage) }\end{array}$ & & & 0988 & 003 & 018 & 042 & 537 & 512 & 047 & 061 & 07 & 091 \\
\hline & UBOCC-A- & Cheese & O. Savary & France & MW81 & MW811 & MW811 & MW811 & MW654 & MW654 & MW811 & MW811 & MW811 & MW811 \\
\hline & $120039^{\mathrm{T}}$ & $\begin{array}{l}\text { (Vacherin } \\
\text { Fribourgeois) }\end{array}$ & & & 0990 & 005 & 020 & 032 & 539 & 514 & 048 & 063 & 078 & 093 \\
\hline
\end{tabular}


Table 1 Continued.

\begin{tabular}{|c|c|c|c|c|c|c|c|c|c|c|c|c|c|c|}
\hline \multirow[t]{2}{*}{ Species } & \multirow[t]{2}{*}{ Isolate } & \multirow[t]{2}{*}{ Substrate } & \multirow{2}{*}{$\begin{array}{l}\text { Collector } \\
\text { and/or } \\
\text { Depositor }\end{array}$} & \multirow[t]{2}{*}{ Locality } & \multicolumn{10}{|c|}{ GenBank Accession No. } \\
\hline & & & & & acl1 & act & cmdA & his3 & ITS & $L S U$ & $r p b 1$ & $r p b 2$ & tef1 & tub2 \\
\hline & $\begin{array}{l}\text { UBOCC-A- } \\
120041\end{array}$ & $\begin{array}{l}\text { Raw cow's } \\
\text { milk cheese }\end{array}$ & O. Savary & France & $\begin{array}{l}\text { MW810 } \\
989\end{array}$ & $\begin{array}{l}\text { MW811 } \\
004\end{array}$ & $\begin{array}{l}\text { MW811 } \\
019\end{array}$ & $\begin{array}{l}\text { MW811 } \\
043\end{array}$ & $\begin{array}{l}\text { MW654 } \\
538\end{array}$ & $\begin{array}{l}\text { MW654 } \\
513\end{array}$ & $\begin{array}{l}\text { MW811 } \\
056\end{array}$ & $\begin{array}{l}\text { MW811 } \\
062\end{array}$ & $\begin{array}{l}\text { MW811 } \\
077\end{array}$ & $\begin{array}{l}\text { MW811 } \\
092\end{array}$ \\
\hline $\begin{array}{l}\text { Longinectria } \\
\text { verticilliforme }\end{array}$ & $\begin{array}{l}\text { UBOCC-A- } \\
120043^{\mathrm{T}}\end{array}$ & $\begin{array}{l}\text { Cheese } \\
\text { (Alpeggio) }\end{array}$ & O. Savary & France & $\begin{array}{l}\text { MW810 } \\
991\end{array}$ & $\begin{array}{l}\text { MW811 } \\
006\end{array}$ & $\begin{array}{l}\text { MW811 } \\
021\end{array}$ & $\begin{array}{l}\text { MW811 } \\
033\end{array}$ & $\begin{array}{l}\text { MW654 } \\
540\end{array}$ & $\begin{array}{l}\text { MW654 } \\
515\end{array}$ & $\begin{array}{l}\text { MW811 } \\
049\end{array}$ & $\begin{array}{l}\text { MW811 } \\
064\end{array}$ & $\begin{array}{l}\text { MW811 } \\
079\end{array}$ & $\begin{array}{l}\text { MW811 } \\
094\end{array}$ \\
\hline
\end{tabular}

Table 2 PCR amplification mix for the ten loci

\begin{tabular}{lllllll}
\hline & $\begin{array}{l}\text { acl1, rpb1, } \\
\text { rpb2, tub2 }\end{array}$ & act & cmdA & his3 & $\begin{array}{l}\text { ITS, } \\
\text { LSU }\end{array}$ & tef1 \\
\hline PCR Buffer $(\mathbf{X})$ & 1 & 1 & 1 & 1 & 1 & 1 \\
MgCl2 $(\mathbf{m M})$ & $1.5^{*}$ & 2 & 2 & 2 & 2 & 1.5 \\
Primers $(\boldsymbol{\mu M})$ & 0.2 & 0.5 & 0.2 & 0.08 & 0.2 & 0.15 \\
dNTPs $(\mathbf{m M})$ & 0.2 & 0.2 & 0.08 & 0.2 & 0.2 & 0.3 \\
GoTaq DNA polymerase $($ Promega) $(\mathbf{U} / \boldsymbol{\mu L})$ & 0.025 & 0.05 & 0.02 & 0.025 & 0.025 & 0.025 \\
\hline
\end{tabular}

*For some isolates, in order to obtain amplicons with rpb1 primers, the $\mathrm{MgCl}_{2}$ concentration was adjusted to $3.5 \mathrm{~mm}$

Phylogenetic analyses were based on Maximum Likelihood (ML) and Bayesian Inference (BI). For ML analysis, the best partition scheme and evolution models were assessed using PartitionFinder2 v2.1.1 using the "greedy" algorithm and selecting the best models for each partition using the corrected Akaike information criterion (AICc), restricting the search among the models available in RAxML2 v8.2.4.1 (Stamatakis 2014) and excluding the models with proportion of invariable (P-Invar) site estimates since it can cause analysis problems according to the RAxML manual (Yang 2006, Stamatakis 2016). The ML analysis was performed using RAxML2 v8.2.4.1 on the ABiMS platform (http://abims.sb-roscoff.fr/), using the default algorithm (-f d) and the GTRGAMMA model. The robustness of the analysis was indirectly evaluated by bootstrap support among 1000 replicates (-N 1000) and a majority-rule consensus tree was further built (-J MR). For BI analysis, the best models (among the namedExtended models of the bModelTest add on in Bayesian Evolutionary Analysis Utility -BEAUti2-) were estimated for each intronic and exonic regions of each gene (a total of 24 partitions) simultaneously with the BI analysis using the Bayesian Evolutionary Analysis Sampling Trees (BEAST)2 v2.6.2 package. The BI analysis was performed using the Monte Carlo Markov Chain method using the BEAST2 v2.6.2 package by performing eight independent repetitions of 100,000,000 generations each, with sampling a tree at every 1,000 generations and a burn-in was fixed at 10\% (discard the first $10 \%$ trees). For both analyses, Allantonectria miltina (CBS 121121) was selected as an outgroup. Convergence of the independent BI analyses was checked using Tracer v1. 7.1. software (Rambaut et al. 2018) and a consensus tree was obtained using Logcombiner and Treeannotator. The two phylogenetic trees obtained with the ML and BI methods were compared using FigTree v1.4.4 (Rambaut 2006) (http://tree.bio.ed.ac.uk/). A tree based on the topology of ML tree with nodes with bootstrap support $<60 \%$ collapsed using Tree Collapser CL 4 (Hodcroft), and annotated with bootstrap and 
posterior probability node supports was built. The number of informative sites was calculated using the web server DIVEIN (Deng et al. 2010). The exact same set of methods was applied to analyze the reduced dataset (six loci) focused on Bisifusarium isolates.

\section{Morphological characters}

Fungal isolates pertaining to putative new species were inoculated in a three-point position on Potato Dextrose Agar (PDA, Difco, Fisher Scientific) for morphological descriptions. Additional cultures were also performed on M2Lev and oatmeal agar $(\mathrm{OA} ; 50 \mathrm{~g} / \mathrm{L}$ oatmeal, $5 \mathrm{~g} / \mathrm{L}$ sodium chloride and $12 \mathrm{~g} / \mathrm{L}$ agar) media and incubated at $25^{\circ} \mathrm{C}$, in the dark. Macroscopic morphological characters (e.g. pigment production, aerial hyphae) were examined after a minimum of 14 days. Moreover, as recommended by Leslie \& Summerell (2006), synthetic nutrient-poor agar (Spezieller Nährstoffarmer Agar, SNA) prepared as described in the publication cited above, was tested for all strains while carrot agar medium $(400 \mathrm{~g} / \mathrm{L}$ carrot, $20 \mathrm{~g} / \mathrm{L}$ agar) was used for the induction of sexual characters when isolates were mated using all possible combinations. Theses plates were incubated at $25^{\circ} \mathrm{C}$, in the dark, for eight weeks.

In parallel, for isolates identified as new type strains, five $\mu \mathrm{L}$ of an inoculum concentrated at $10^{6}$ conidia/mL were also inoculated on PDA medium (two biological and two technical replicates) and incubated at 15 and $25^{\circ} \mathrm{C}$ in darkness. Colony diameters were monitored for 40 and 21 days, respectively. Growth rates fitting each kinetic were estimated by the modified Gompertz equation (Zwietering et al. 1990) and using the MATLAB software (MATLAB. 9.5.0.944444. (R2018b), Natick, Massachusetts: The MathWorks Inc.). Two supplementary technical replicates were also deposited and incubated at 5,10,20,30 and $37^{\circ} \mathrm{C}$ in darkness, then colony diameters were monitored during 14 days to determine growth limits as a function of temperature.

As for microscopic characteristics, descriptions were done based on PDA cultures as well as supplementary observations on M2Lev medium after 7 to 17 days growth. Fungal structures were mounted on glass slides using lactophenol cotton blue solution as a mounting fluid. Additional observations were performed after 40-46 days (fourteen days in the dark then exposure to natural light during a minimum of fourteen days) to potentially observe chlamydospores. An Olympus BX40 microscope equipped with an Olympus Digital Camera DP70 (Olympus, Tokyo, Japan) was used to observe microscopic characteristics (e.g. presence, shape and length of macroconidia and/or microconidia, phialidic pegs, chlamydospores) based on the recommendation of "The Fusarium laboratory manual" (Leslie \& Summerell 2006). Several measurements were taken with a magnification of 400x and 1000x.

Then, conidia observations were performed on the isolates identified as new type strains using scanning electron microscopy. To do so, two $\mathrm{mL}$ of tween $80(0.005 \%$, v/v) were added on each $10-$ 12 days monospore culture on PDA medium to collect conidia suspensions. Concentrations were determined by counting spores in Malassez cells and were adjusted at $10^{7}$ conidia per $\mathrm{mL}$. One $\mathrm{mL}$ of each suspension was filtered on a $0.2 \mu \mathrm{m}$ polycarbonate membrane filter (GTTP01300, Milipore, Merck, Germany) using a 13 mm filter holder (SX0001300, Milipore, Merck, Germany). The filter was transferred on a Whatman filter paper to absorb excess liquid, then a fragment was bonded on the observation plot. Finally, two $\mu$ L of Hitachi ionic liquid HILEM IL 1000 (Hitachi High-Tech, Europe, $\mathrm{GmbH}$ ) was deposited and the observations were directly performed by scanning electron microscopy (SEM; HITACHI S-3200N) at the PIMM microscopy platform (Université de Bretagne Occidentale, France), without any additional fixation or metallization steps.

\section{Secondary metabolite production}

The four holotype strains UBOCC-A-120021, UBOCC-A-120036, UBOCC-A-120039 and UBOCC-A-120043 were inoculated as three replicates using five $\mu \mathrm{L}$ of $10^{6}$ conidia/mL suspensions, on PDA, malt extract agar (MEA: $20 \mathrm{~g} / \mathrm{L}$ malt extract, $1 \mathrm{~g} / \mathrm{L}$ peptone, $20 \mathrm{~g} / \mathrm{L}$ glucose and $20 \mathrm{~g} / \mathrm{L}$ agar), yeast extract sucrose agar (YES, pH 5.2) and skim milk agar (100 g/L Difco skim milk powder, $10 \mathrm{~g} / \mathrm{L}$ yeast extract and $20 \mathrm{~g} / \mathrm{L}$ agar) for 14 days at $25^{\circ} \mathrm{C}$. Then, five $6 \mathrm{~mm}$ plugs were removed from the agar colonies for extractions with ethyl acetate / isopropanol (3:1) supplemented with 1\% 
(vol/vol) formic acid using ultra-sonication. After removal of the extracted plugs, the remaining liquid was evaporated and the extract re-dissolved in methanol. Three $\mu \mathrm{L}$ of the methanol extract were analyzed by high performance liquid chromatography (HPLC) using diode array (DAD) and fluorescence detection (emission $230 \mathrm{~nm}$, excitation $333 \mathrm{~nm} \&$ emission $230 \mathrm{~nm}$ and excitation 450 nm) (Thermo Scientific, Dionex, Sunnyvale, California, USA) as described by Frisvad \& Thrane (1987). A bracketed alkylphenone retention index was calculated for each compound as described by Frisvad \& Thrane (1987, 1993). The UV spectra of the compounds eluting were compared to authentic standards of known secondary metabolites as described by Nielsen et al. (2011), Klitgaard et al. (2014) and Kildgaard et al. (2014) by comparing retention time, retention index and UV spectra recorded from $200-600 \mathrm{~nm}$ (diode array detection). The water / acetonitrile gradient used in the HPLC analyses and the column and other conditions used are described by Nielsen et al. (2011).

\section{Results}

\section{Cheese sampling and analysis}

Among the 56 cheeses sampled to collect $B$. domesticum isolates, a large number of fungal isolates pertaining to different genera such as Geotrichum, Mucor, Penicillium or Scopulariopsis were observed (data not shown) along with the typical B. domesticum morphotypes ( $\mathrm{n}=9$, one isolate per cheese). Interestingly, other isolates ( $\mathrm{n}=10$, one macroscopically different isolate per cheese) exhibited Fusarium-like morphotypes. Identifications based on translation elongation factor 1-alpha sequencing confirmed that the isolates exhibiting the typical $B$. domesticum morphotypes indeed belonged to the latter species, while the sequences obtained for the Fusarium-like isolates could not be identified at the species level. Based on the observed blastn scores (closest identification to already described Nectriaceae species estimated to 83.5 to $85.9 \%$, data not shown), the possibility of four putative new Nectriaceae species was highlighted. Among these isolates, two were macroscopically similar to an environmental strain (surface of sandstone building) described as Trichothecium sp. (94.9\% similarity to B. domesticum based on ITS gene (Suihko et al. 2007)). ITS sequencing later confirmed the identity of this environmental strain with two of the obtained cheese isolates (data not shown), which was therefore included in this phylogenetic analysis (for a total of 11 unidentified Fusarium-like isolates). Noteworthy, during cheese investigation, other Nectriaceae species were identified, namely $F$. babinda (e.g. UBOCC-A-120044), F. oxysporum (e.g. ESE 00175), $F$. culmorum (e.g. ESE 00152) and $F$. solani s.l. (e.g. UBOCC-A-120045, ESE 00026, ESE 00073, ESE 01219), indicating that, beyond $B$. domesticum, members of this family can be associated with this food matrix although they may well be just environmental contaminants.

\section{Phylogenetic analyses}

A phylogenetic reconstruction (Fig. 1) was performed using a concatenated non-redundant dataset of ten loci: acl1, act, cmdA, his3, ITS, LSU, rpb1, rpb2, tef1 and tub2 sequences of 64 strains (Table 1, Supplementary Table 2) belonging to 19 genera (18 based on Lombard et al. nomenclature (2015) and a new genus proposed in this study), as well as A. miltina CBS 121121 as an outgroup. The multi-gene alignment length was 5134 nucleotides, including few gaps and missing data (acll 487 bp, act 536 bp, cmdA 413 bp, his3 319 bp, ITS 533 bp, LSU 758 bp, rpbl 656 bp, rpb2 780 bp, tefl $351 \mathrm{bp}$ and tub2 301bp). Moreover, this alignment contained 2226 informative sites (2122 without gaps). After alignment using the ten loci dataset, some of the unidentified isolates presented high identity levels between them, thus forming four distinct genetic groups, namely (i) UBOCC-A120021, UBOCC-A-120034 and VTT-D-041022 (maximum divergence of 10/6441 nucleotides over the whole raw dataset), (ii) UBOCC-A-120035, UBOCC-A-120036 and UBOCC-A-120037 (maximum divergence of 2/6173 nucleotides), (iii) ESE-00140, UBOCC-A-120038, UBOCC-A120039 and UBOCC-A-120041 (maximum divergence of 6/6187 nucleotides) and (iv) UBOCC-A120043. Therefore, given the very low nucleotide divergence, only one representative sequence for each group, namely UBOCC-A-120021, UBOCC-A-120036, UBOCC-A-120039 and UBOCC-A120043, was conserved for further phylogenetic analyses. The obtained phylogenetic tree (Fig. 1) 


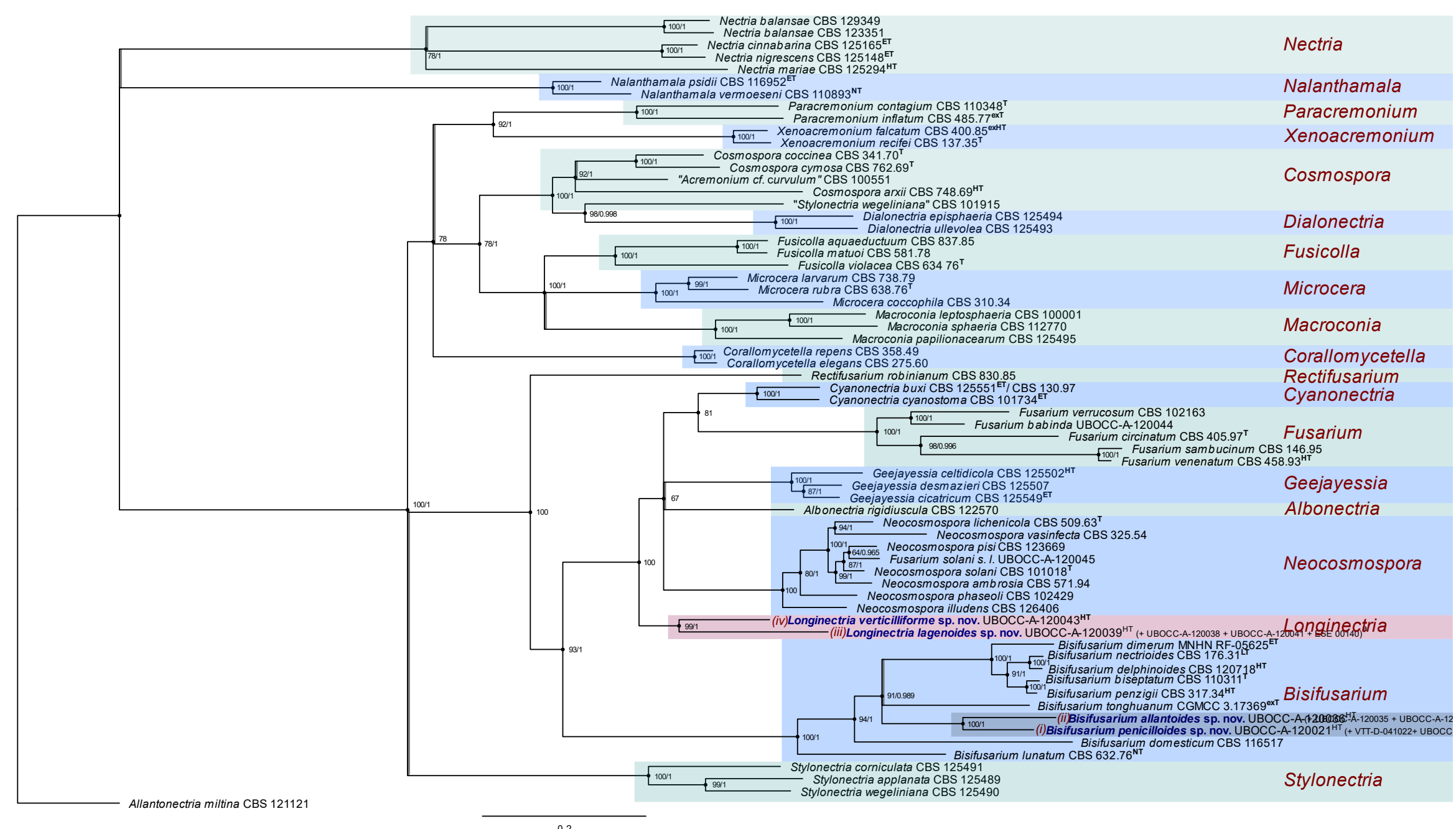

Figure 1 - Maximum-Likelihood phylogenetic consensus tree of Nectriaceae species inferred from the combined ten gene sequence subset (acll, act, cmdA, his3, ITS, LSU, rpb1, rpb2, tef1 and tub2). Maximum-Likelihood bootstrap supports (in percentage, 1000 replicates) and Bayesian posterior probabilities are represented at nodes (respectively right and left). The tree is rooted with A. miltina CBS 121121. "ET", "LT", "HT", "T", "exT", "exHT", "NT" respectively mean epitype, lectotype, holotype, type, ex-type, ex-holotype and neotype. The four new species are written in blue and bold. "a": Only the sequences of the type strain of each proposed species were used in the phylogenetic analysis given the very low nucleotide divergence between strains within the considered species. 
indicated that the putative new species were not clustered with already described species within the Nectriaceae family. Interestingly, UBOCC-A-120021 and UBOCC-A-120036 were nested within the Bisifusarium genus (Fusarium dimerum species complex sensu Geiser et al. 2021) with nucleotide differences comprised between 566 to 676/5134 and 571 to 680/5134, respectively, with other already described Bisifusarium species (Supplementary Table 3) and therefore likely correspond to two new species within this genus. On the other hand, UBOCC-A-120039 and UBOCC-A-120043 constituted an independent sister clade with a group including different Nectriaceae genera (described by Lombard et al. (2015) as Neocosmospora, Albonectria, Geejayessia, Cyanonectria and Fusarium) with a minimum nucleotide divergence of 566/5134 bp with species from these clades (Supplementary Table 4) suggesting that they belong to a distinct genus from Neocosmospora, Albonectria, Geejayessia, Cyanonectria and Fusarium, and other more phylogenetically distant Nectriaceae. Indeed, this minimum nucleotide divergence are comparable to those observed between the genera within this group, or even higher as for Neocosmospora and Albonectria (539/5134 bp) or Neocosmospora and Geeyassia (560/5134 bp) or Neocosmospora and Cyanonectria (554/5134 bp) (Supplementary Table 4).

As the latest addition to the Bisifusarium genus (B. tonghuanum) was based only on six genes of the "Lombard et al." gene set, we wanted to confirm that the missing data had no impact on the positioning of putative Bisifusarium isolates. Therefore, a second phylogenetic reconstruction was performed using a concatenated non-redundant dataset of the six common loci (ITS, LSU, rpb1, rpb2, tefl and tub2) of the ten species belonging to the Bisifusarium genus (the eight already described and the two additional ones based on this study) and $F$. venenatum CBS $458.93^{\mathrm{T}}$ as an outgroup. This alignment length was 2854 nucleotides long, including few gaps. It contained 400 informative sites (391 without gaps). The resulting ML and BI analyses were highly concordant (data not shown) and the position of UBOCC-A-120021 and UBOCC-A-120036 in the resulting phylogenetic tree (Fig. S1) was concordant with the one observed for the ten loci-based analysis. Bisifusarium allantoides sp. nov. and B. penicilloides sp. nov. were placed in a sister clade of $B$. tonghuanum with a nucleotide divergence of 225 and 193/2854 nucleotides, respectively with B. tonghuanum CGMCC 3.17369. Both multi-gene phylogenetic analyses were deposited in TreeBASE (Study ID 28488 and 28538).

\section{Taxonomy}

Bisifusarium allantoides O. Savary, M. Coton, E. Coton and J-L. Jany, sp. nov.

Figs 2, 3

MycoBank number: MB840947

Etymology - From latin "allantois" referring to the allantoid-shape of the conidia.

Holotype - UBOCC-A-120036, CBS 147587

Macromorphology - Based on UBOCC-A-120036 (CBS 147587) colony growth for 16 days at $25^{\circ} \mathrm{C}$, colony surface on PDA was pale orange (RGB $[167,137,106]$ to $[175,137,90]$ ) with many white powdery structures and cottony hyphae, more or less aerial, with an elevation at the colony center (Fig. 2A). Margins were smooth and less colored. After natural light exposure during several days, colony color became more pronounced. It can be noted that the mycelium was very well attached to the agar. The colony reverse on PDA was orange at the center (RGB [148, 100, 59]) and progressively less colored until the margins (RGB [144, 130, 103]) (Fig. 2B). No pigment diffusion in the agar was observed. Interestingly, on the M2Lev medium, the morphological aspect was highly similar to that on PDA with more cottony structures, a more pronounced colony color, and smooth and regular margins (Fig. 3).

Micromorphology - Ascomatal state unknown. Phialides were less abundant than in the other described Bisifusarium species. Monophialides formed terminally (Fig. 2C) or laterally on hyphae and were cylindrical, tapering toward the tip (81-127 $\mu \mathrm{m})$. Formation of lateral phialidic pegs common on PDA (and on M2Lev) were observed (Fig. 2D). Polyphialides were not observed. Microconidia and macroconidia were difficult to distinguish (Fig. 2E-K) but observed macroconidia were typically allantoidal, straight or curved and cylindrical (size on average $12.41 \times 3.2 \mu \mathrm{m}, 9.5-$ $23.6 \times 2.72-4.1 \mu \mathrm{m})$. The apical cells were papillate while the basal cells were distinctly notched. 
They were mostly 1-septate or more. Microconidia could be characterized by short conidia 0 -septate or 1-septate with median septum or mostly an off-centered septum (size on average $7.6 \times 2.5 \mu \mathrm{m}$, 4.7-9.7 x 1.5-3.2 $\mu \mathrm{m}$ ). They were cylindrical, straight or curved, and slightly notched. After several weeks of growth, no chlamydospore was observed. SNA medium did not provide any supplementary information.

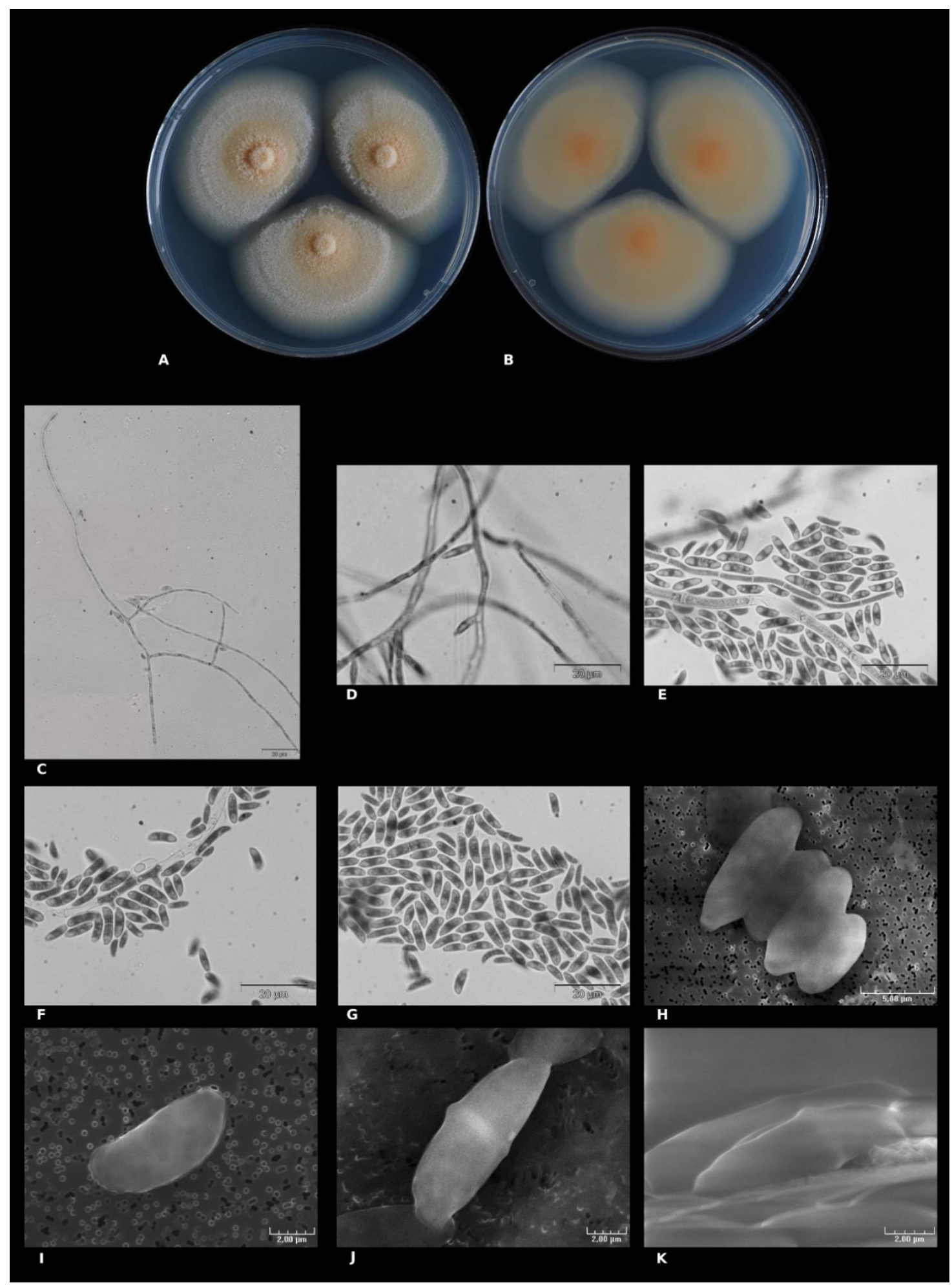

Figure 2 - Macroscopic morphological and microscopic characteristics of Bisifusarium allantoides (UBOCC-A-120036 $\left.{ }^{\mathrm{HT}}\right)$. A colony on PDA $\left(14\right.$ days at $\left.25^{\circ} \mathrm{C}\right)$. B colony reverse on PDA (14 days at $25^{\circ} \mathrm{C}$ ). $\mathrm{C}$ terminal monophialides. D phialidic pegs. $\mathrm{E}-\mathrm{K}$ conidia. $\mathrm{C}-\mathrm{G}$ microscopic acquisitions using classical microscopy. $\mathrm{H}-\mathrm{K}$ microscopic acquisition using scanning electron microscopy. 
Growth characteristics on PDA - Moderately fast growth was observed at $25^{\circ} \mathrm{C}$, colony diameter measuring $70.5 \pm 1.7 \mathrm{~mm}$ versus $43.5 \pm 0.6 \mathrm{~mm}$ at $15^{\circ} \mathrm{C}$ and growth rates were estimated at $5.2 \pm 0.3 \mathrm{~mm} /$ days versus $3.3 \pm 0.1 \mathrm{~mm} /$ days, respectively. Moreover, $B$. allantoides growth was observed between $5^{\circ} \mathrm{C}$ and $30^{\circ} \mathrm{C}$, colony diameters measuring at 14 days were $8.0 \pm 0.0 \mathrm{~mm}$ at $5^{\circ} \mathrm{C}$, $19.0 \pm 0.0 \mathrm{~mm}$ at $10^{\circ} \mathrm{C}, 55.0 \pm 4.2 \mathrm{~mm}$ at $20^{\circ} \mathrm{C}, 39.0 \pm 2.8 \mathrm{~mm}$ at $30^{\circ} \mathrm{C}$ and no growth at $37^{\circ} \mathrm{C}$.

Secondary metabolites - No known mycotoxins already described to be produced by Fusarium, Penicillium, Aspergillus or Alternaria spp. (e.g. fusaric acid, zearalenone, trichothecenes, butenolide or enniatins) were detected. For most detected compounds, except ergosterol, extrolites could not be identified but belonged to 47 chromophore families (Table 3). These secondary metabolites included the "emon" chromophore or corresponded to extra apolar free fatty acids (including linoleic acid and probably oleic acid), mid-cyclic lipopeptides with tyrosine in the cyclic peptide, indole alkaloids or 2-pyruvoylaminobenzamide-like molecules (Table 3).

Substrate and distribution - To date, B. allantoides has been solely isolated from European cheese.

Material examined - France, isolated from French soft cheese rind, F. Deniel, 11 Dec. 2013, holotype UBOCC-A-120036 = CBS 147587.

Additional material examined - France, isolated from French soft cheese rind, F. Deniel, UBOCC-A-120037, 11 Dec. 2013; Spain, isolated from Spanish cheese (Manchego), B. Mayo, before Nov. 2018, UBOCC-A-120035.

Note - On oatmeal agar media, sporodochia could be noted for the three $B$. allantoides strains (Fig. 3).

Bisifusarium penicilloides O. Savary, M. Coton, E. Coton and J-L. Jany, sp. nov.

Figs 3, 4

MycoBank number: MB840948

Etymology - From latin "penicillus", referring to the penicillate organisation of polyphialides.

Holotype - UBOCC-A-120021, CBS 147586

Macromorphology - Based on UBOCC-A-120021 (CBS 147586) colony growth for 16 days at $25^{\circ} \mathrm{C}$ on PDA, short aerial mycelial strands with a fungal colony very well attached to the agar. Colony surface on PDA was slightly colored, beige (RGB $[191,181,163]$ ) with yellow shades (Fig. 4A) and color intensified during sporulation in natural light becoming orange (RGB [210,163,114]). No powdery structure was observed, but velvety and downy aspects were. A slight elevation could be noted in the center and lighter regular margins were observed. The colony reverse on PDA was pale (RGB [168,163,149]) (Fig. 4B), to orange pale (RGB [170,143,119) after natural light exposure for several days. No pigment production in agar was observed. On M2Lev medium (Fig. 3), a similar macroscopic profile was observed with slightly more aerial hyphae and more pronounced colony color than on PDA medium.

Micromorphology - Ascomatal state unknown. Several types of conidiogenous cells were observed. Indeed, while polyphialides with a penicillate configuration were frequently observed (Fig. 4C, D) (phialides length after bifurcation on average $15 \mu \mathrm{m}, 9-25 \mu \mathrm{m}$ ) and appear as a species characteristic. several monophialides formed terminally $(\geq 73-92 \mu \mathrm{m})$ or laterally on hyphae and lateral phialidic pegs (Fig. 4E) could be also noted. Phialides were cylindrical tapering towards the tip. Microconidia were not observed and macroconidia were mostly 0 -septate or rarely 1 -septate with a median septum (Fig. 4F-J) (size on average $6.5 \times 2.7 \mu \mathrm{m}, 5.6-7.2 \times 2.1-3.2 \mu \mathrm{m}$ ). They were ellipsoidal, straight or curved presenting a lunate or reniform shape, and basal cells were distinctly notched. After several weeks of growth, globose chlamydospores were observed, typically intercalary, solitary or in chains that can be long. SNA medium did not provide any supplementary information.

Growth characteristics on PDA - Slow growth was observed at $25^{\circ} \mathrm{C}$, colony diameter measuring $36.0 \pm 1.8 \mathrm{~mm}$ versus $43.0 \pm 0.8 \mathrm{~mm}$ at $15^{\circ} \mathrm{C}$ and the growth rates were estimated at 2.6 $\pm 0.2 \mathrm{~mm} /$ days versus $3.2 \pm 0.1 \mathrm{~mm} /$ days, respectively. Moreover, B. penicilloides growth was observed between $5^{\circ} \mathrm{C}$ and $25^{\circ} \mathrm{C}$, colony diameters measuring at 14 days $9.5 \pm 0.7 \mathrm{~mm}$ at $5^{\circ} \mathrm{C}, 20.5$ $\pm 0.7 \mathrm{~mm}$ at $10^{\circ} \mathrm{C}, 49.0 \pm 1.4 \mathrm{~mm}$ at $20^{\circ} \mathrm{C}$, and no growth at $30^{\circ} \mathrm{C}$ and $37^{\circ} \mathrm{C}$. 


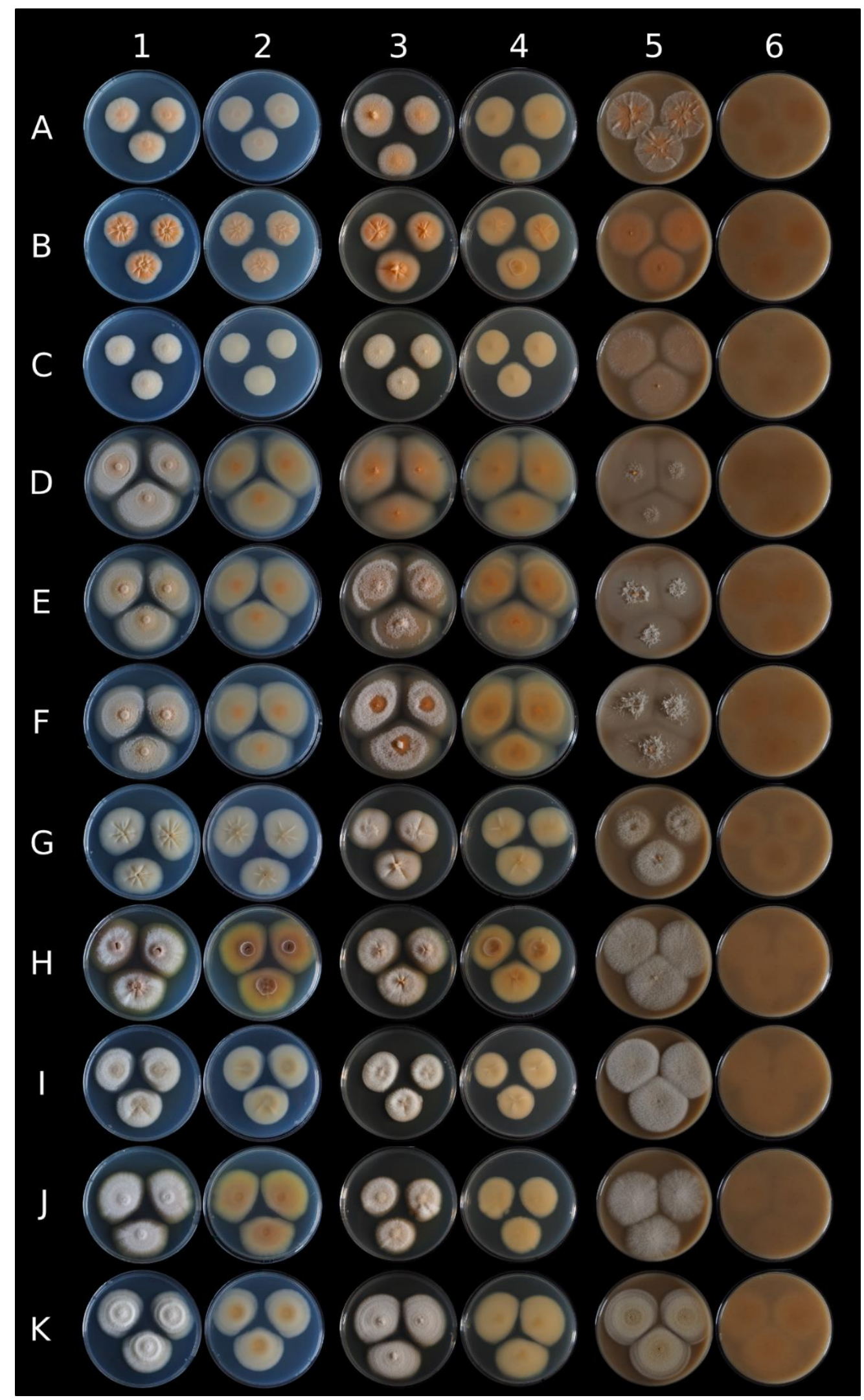

Figure 3 - Macroscopic comparison of eleven strains belonging to four new Nectriaceae species on different culture media. Lines A to K respectively correspond to Bisifusarium penicilloides VTT-D041022, B. penicilloides UBOCC-A-120034, B. penicilloides UBOCC-A-120021, Bisifusarium allantoides UBOCC-A-120035, B. allantoides UBOCC-A-120036, B. allantoides UBOCC-A120037, Longinectria lagenoides UBOCC-A-120038, L. lagenoides UBOCC-A-120039, L. lagenoides UBOCC-A-120041, L. lagenoides ESE-00140 and Longinectria verticilliforme 
UBOCC-A-120043 grown during 14 days at $25^{\circ} \mathrm{C}$ on Potatoes Dextrose Agar (column $1 \&$ 2: observe \& reverse), M2Lev (column $3 \&$ 4: observe \& reverse) and oatmeal agar (column 5 \& 6: observe \& reverse).

Secondary metabolites - No known mycotoxins already described to be produced by Fusarium, Penicillium, Aspergillus or Alternaria spp. were detected. For most detected compounds, except ergosterol, extrolites could not be identified but belonged to 47 chromophore families (Table 3). These secondary metabolites included the "emon" chromophore, extra apolar free fatty acids (including linoleic acid and probably oleic acid), mid-cyclic lipopeptides with tyrosine in the cyclic peptide, indole alkaloids or 2-pyruvoylaminobenzamide-like molecules (Table 3).

Substrate and distribution - Two strains isolated from cheese rind, including the holotype. One strain (VTT-D-041022), previously identified as Trichothecium sp. (94.9\% similarity to B. domesticum, based on ITS sequencing) was also obtained from the outer surface of a sandstone building, Arbroat Abbey (Suihko et al. 2007).

Material examined - France, isolated from French soft cheese (Mont d'Or), O. Savary, 22 Jan. 2019, holotype UBOCC-A-120021 = CBS 147586.

Additional material examined - France, isolated from French cheese (Mont d'Or), O. Savary, 21 Jan. 2019, UBOCC-A-120034; Scotland, isolated from outer surface of sandstone building, Arbroat Abbey, Suihko et al. (2007), before Nov. 2006, VTT-D-041022.

Note - A "yeasty" odour was clearly detected during fungal growth on PDA. A few macroscopic differences were observed among the three studied isolates (Fig. 3). Colony surface was more colorful during the first days of culture for the VTT-D-041022 and UBOCC-A-120034 isolates but after several days of exposure to natural light, all three fungal cultures presented the same intense color. While VTT-D-041022 and UBOCC-A-120021 had the same mycelium characteristics, UBOCC-A-120034 strain colonies were radially furrowed with a concave center and margins were lighter and irregular.

Longinectria O. Savary, M. Coton, E. Coton and J-L. Jany, gen. nov.

MycoBank number: MB840949

Etymology - From the latin longus = long, "Longi-" refers to the phialides length (on average $42 \mu \mathrm{m} ; 15-72 \mu \mathrm{m}$, some being very long - 153 to $237 \mu \mathrm{m}$ ) observed for the Longinectria species and "-nectria" refers to the Nectriaceae family.

Type species - Longinectria lagenoides O. Savary, M. Coton, E. Coton \& J-L. Jany, sp. nov.

Ascomatal state unknown. Conidiophores are variable-length phialides, sometimes extremely long (e.g. 153-237 $\mu \mathrm{m}$ ), lateral, sometimes verticillate, hyaline. Macroconidia straight to slightly curved, apical cell morphology blunt to papillate and a basal cell often notched, 0-3 septate, hyaline. Microconidia ovoid, ellipsoid to allantoid, 0-1 septate, hyaline. Chlamydospores absent to abundant, globose, single, in pairs or chains, intercalary or terminal.

Longinectria lagenoides O. Savary, M. Coton, E. Coton and J-L. Jany, sp. nov.

Figs 3, 5

MycoBank number: MB840950

Etymology - From latin lagoena $=$ bottle, refers to the observed phialide shape.

Holotype - UBOCC-A-120039, CBS 147588

Macromorphology - Based on UBOCC-A-120039 (CBS 147588), colony growth for 16 days at $25^{\circ} \mathrm{C}$, colony surface on PDA was heterogeneously colored (Fig. 5A). The center was mainly brown (RGB [139,112,93]), the smooth margin was brown (RGB [104,95,78]) and intensified during growth. The intermediate zone appeared grey (RGB [164,167,173]) with brown shades because of the aerial white hyphae with powdery structures. Brown pigments were diffused in the agar around the colony. Colonies were radially furrowed with a convex center. Colony reverse was also heterogeneously colored (Fig. 5B), the center being brown (RGB [98,61,43]), then progressively clearer (RGB [128,90,51]) and highly visible on the reverse face, the fungal colony dug into the agar. Concerning fungal growth on M2Lev, colonies were beige with shades of brown and, in particular, 
in the regular margins and convex center (Fig. 3). Very aerial hyphae were observed with powdery and/or cottony structures and the mycelium dug into agar.

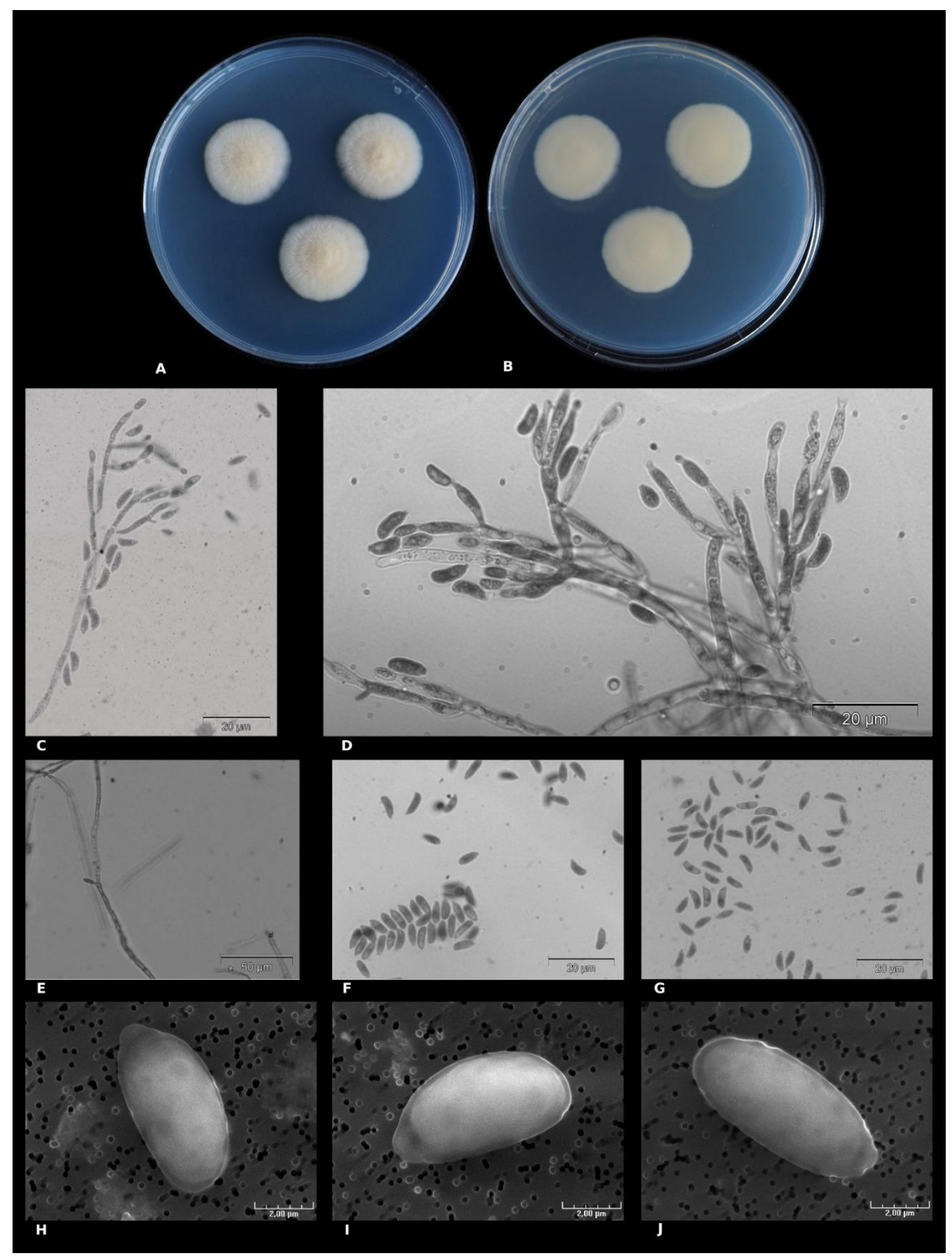

Figure 4 - Macroscopic morphological and microscopic characteristics of Bisifusarium penicilloides (UBOCC-A-120021 $\left.{ }^{\mathrm{HT}}\right)$. A Colony on PDA $\left(14\right.$ days at $\left.25^{\circ} \mathrm{C}\right)$. B Colony reverse on PDA (14 days at $25^{\circ} \mathrm{C}$ ). $\mathrm{C}-\mathrm{D}$ polyphialides. E phialidic pegs. $\mathrm{F}-\mathrm{J}$ conidia. $\mathrm{C}-\mathrm{G}$ microscopic acquisitions using classical microscopy. H-J microscopic acquisitions using scanning electron microscopy.

Micromorphology - Ascomatal state unknown. Lateral phialides with different sizes were observed (on average $44 \mu \mathrm{m}, 15-72 \mu \mathrm{m}$ ), some being very long (size 153-237 $\mu \mathrm{m}$ ) (Fig. 5C-D) 
while others are short and could even be assimilated to phialidic peg. Macroconidia 0-3 septate, very straight in shape with an apical form, blunt to papillate, and basal cell very pronounced and notched (Fig. 5E -5J). Their size is on average 17 x $4 \mu \mathrm{m}(12-29 \times 3-7 \mu \mathrm{m})$ length range. Microconidia 0-1 septate ovoid to allantoid shape (8-12 x 3-5), apical form blunt and basal cell slightly notched (Fig. $5 \mathrm{E}, \mathrm{G})$. After several weeks of growth, globoses chlamydospores were observed typically intercalary, or terminal with mainly $\geq 2$ chlamydospores. SNA medium did not provide any supplementary information.

Growth characteristics on PDA - Slow growth was observed at $25^{\circ} \mathrm{C}$, colony diameter measuring $53.0 \pm 1.6 \mathrm{~mm}$ versus $32.3 \pm 0.5 \mathrm{~mm}$ at $15^{\circ} \mathrm{C}$ and the growth rates were estimated at 3.7 $\pm 0.1 \mathrm{~mm} /$ days versus $2.3 \pm 0.0 \mathrm{~mm}$ /days, respectively. Moreover, L. lagenoides growth was observed between $5^{\circ} \mathrm{C}$ and $25^{\circ} \mathrm{C}$, colony diameters measuring at 14 days $4.5 \pm 0.0 \mathrm{~mm}$ at $5^{\circ} \mathrm{C}, 13.0$ $\pm 0.0 \mathrm{~mm}$ at $10^{\circ} \mathrm{C}, 44.5 \pm 2.1 \mathrm{~mm}$ at $20^{\circ} \mathrm{C}$, and no growth at $30^{\circ} \mathrm{C}$ and $37^{\circ} \mathrm{C}$.

Secondary metabolites - No known mycotoxins already described to be produced by Fusarium, Penicillium, Aspergillus or Alternaria spp. were detected. For most detected compounds, except ergosterol, extrolites could not be identified but belonged to 38 chromophore families (Table 3). These secondary metabolites again included the "emon" chromophore or corresponded to extra apolar free fatty acids (including linoleic acid and probably oleic acid), mid-cyclic lipopeptides with tyrosine in the cyclic peptide, indole alkaloids, 2-pyruvoylaminobenzamide-like molecules but also an alkylphenone chromophore and atrovenetin chromophore (Table 3).

Substrate and distribution - To date, L. lagenoides has been solely isolated from European cheese.

Material examined - France, isolated from Swiss cheese, O. Savary, 22 Nov. 2018, holotype UBOCC-A-120039, CBS 147588.

Additional material examined - France, isolated from Swiss cheese, O. Savary, 20 Nov. 2018, UBOCC-A-120038; France, isolated from Swiss cheese, O. Savary, UBOCC-A-120041, 27 Nov. 2018; France, isolated from semi-hard Swiss cheese, J. Ropars, ESE 00140, 2018.

Note - Few macroscopically differences were observed among the four isolates observed on PDA (Fig. 3). Indeed, colony color was more or less pronounced (RGB [156,153,136] or $[157,157,162])$ depending on the considered strain), and in particular, one strain (UBOCC-A120038) remained yellow/beige (RGB $[151,153,148]$ ) even with natural light exposure during several days. Radial furrows with a convex center were mainly pronounced for UBOCC-A-120038 and UBOCC-A-120039 but also slightly more observable for ESE-00140. The colony reverse could be beige (RGB [140,134,121]) to brown (RGB [143,109,80]) depending on the strains.

Longinectria verticilliforme O. Savary, M. Coton, E. Coton and J-L. Jany, sp. nov.

Figs 3, 6

MycoBank number: MB840951

Etymology - Name refers to the phialides bifurcation as a verticille.

Holotype - UBOCC-A-120043, CBS 147589

Macromorphology - Based on UBOCC-A-120043 (CBS 147589) colony growth for 16 days at $25^{\circ} \mathrm{C}$, colony surface on PDA was white (RGB [209,212,218]) to white grey (RGB $[176,175,178]$ ) (Fig. 6A) and did not change color after several days when exposed to natural light. Mycelium was very powdery and cottony, with more elevated circular zones of slightly aerial hyphae. Interestingly, few transparent exudate droplets have been observed at $25^{\circ} \mathrm{C}$ as well as at $15^{\circ} \mathrm{C}$. Colony reverse on PDA was more colored in the center (RGB $[153,124,85]$ ) then lighter until the margin (RGB [147,141,129]) (Fig. 6B). No pigment production in agar was observed. Moreover, a powderier aspect was observed on M2Lev medium (Fig. 3) versus PDA and the margins, although smooth and regular, were beige instead of grey white.

Micromorphology - Ascomatal state unknown. Verticillate phialides (mainly three branches or more) which could be in terminal as well lateral position with successive bifurcations were observed (Fig. 6C-E). Lateral phialides were also noted, sometimes very long (on average, length after bifurcation: $39 \mu \mathrm{m}$, with variations from 27 to $58 \mu \mathrm{m}$ ), and frequently septate containing a probable formation of new phialide bifurcations (size 3-15 $\mu \mathrm{m}$ ). Moreover, conidia clusters, similar to false 
head structures, formed from the same isolated phialides were observed (Fig. 6F). Macroconidia 13 septate, mainly straight or slightly ellipsoidal with an apical cell morphology blunt to papillate and a basal cell distinctly notched, size on average $15.3 \times 2.8 \mu \mathrm{m}(9.4-26.9 \times 2.3-3.2 \mu \mathrm{m})$ (Fig. 6G-J). Microconidia 0-1 septate, straight or curved, reniform, apical cells morphology is blunt while basal cell is notched, size on average 7.6-3.1 (5.5-9.5 x 2.0-4.1 $\mu \mathrm{m})$ (Fig. 6I, K). No chlamydospores observed. SNA medium did not provide any supplementary information.

Growth characteristics on PDA - Slow growth was observed at $25^{\circ} \mathrm{C}$, colony diameter measuring $49.5 \pm 3.3 \mathrm{~mm}$ versus $28.6 \pm 2.9 \mathrm{~mm}$ at $15^{\circ} \mathrm{C}$ and the growth rates were estimated at 3.4 $\pm 0.3 \mathrm{~mm} /$ days versus $2.1 \pm 0.3 \mathrm{~mm} /$ days, respectively. Moreover, L. verticilliforme growth was observed between $5^{\circ} \mathrm{C}$ and $25^{\circ} \mathrm{C}$, colony diameters measuring at 14 days $5.0 \pm 0.0 \mathrm{~mm}$ at $5^{\circ} \mathrm{C}, 15.0$ $\pm 0.0 \mathrm{~mm}$ at $10^{\circ} \mathrm{C}, 41.5 \pm 0.7 \mathrm{~mm}$ at $20^{\circ} \mathrm{C}$, and no growth at $30^{\circ} \mathrm{C}$ and $37^{\circ} \mathrm{C}$.

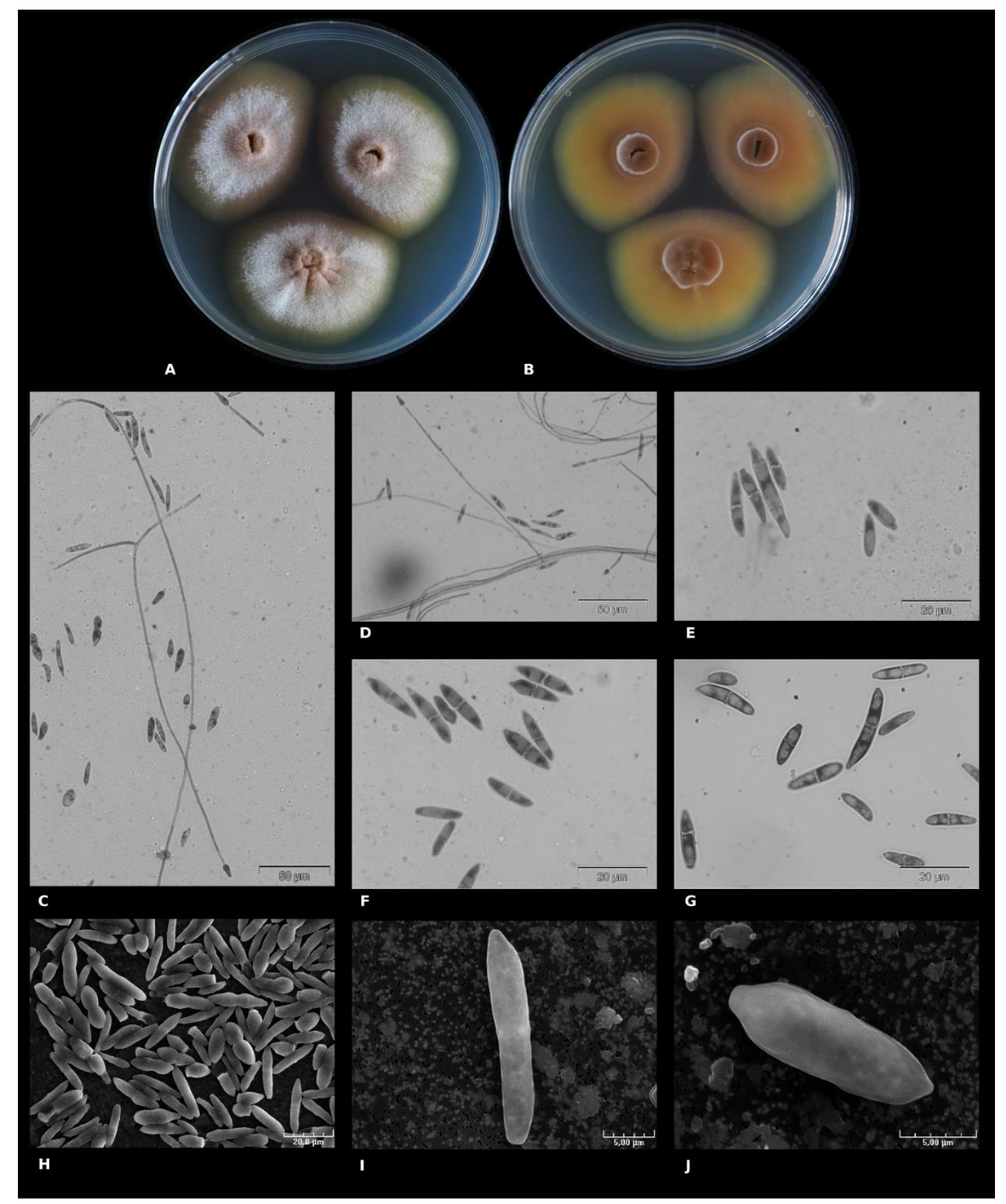

Figure 5 - Macroscopic morphological and microscopic characteristics of Longinectria lagenoides (UBOCC-A-120039 ${ }^{\mathrm{HT}}$ ). A Colony on PDA (14 days at $\left.25^{\circ} \mathrm{C}\right)$. B Colony reverse on PDA (14 days at 
$25^{\circ} \mathrm{C}$ ). C-D phialides. E-J micro- and macro- conidia. $\mathrm{C}-\mathrm{G}$ microscopic acquisitions using classical microscopy, $\mathrm{H}-\mathrm{J}$ : microscopic acquisition using scanning electron microscopy.

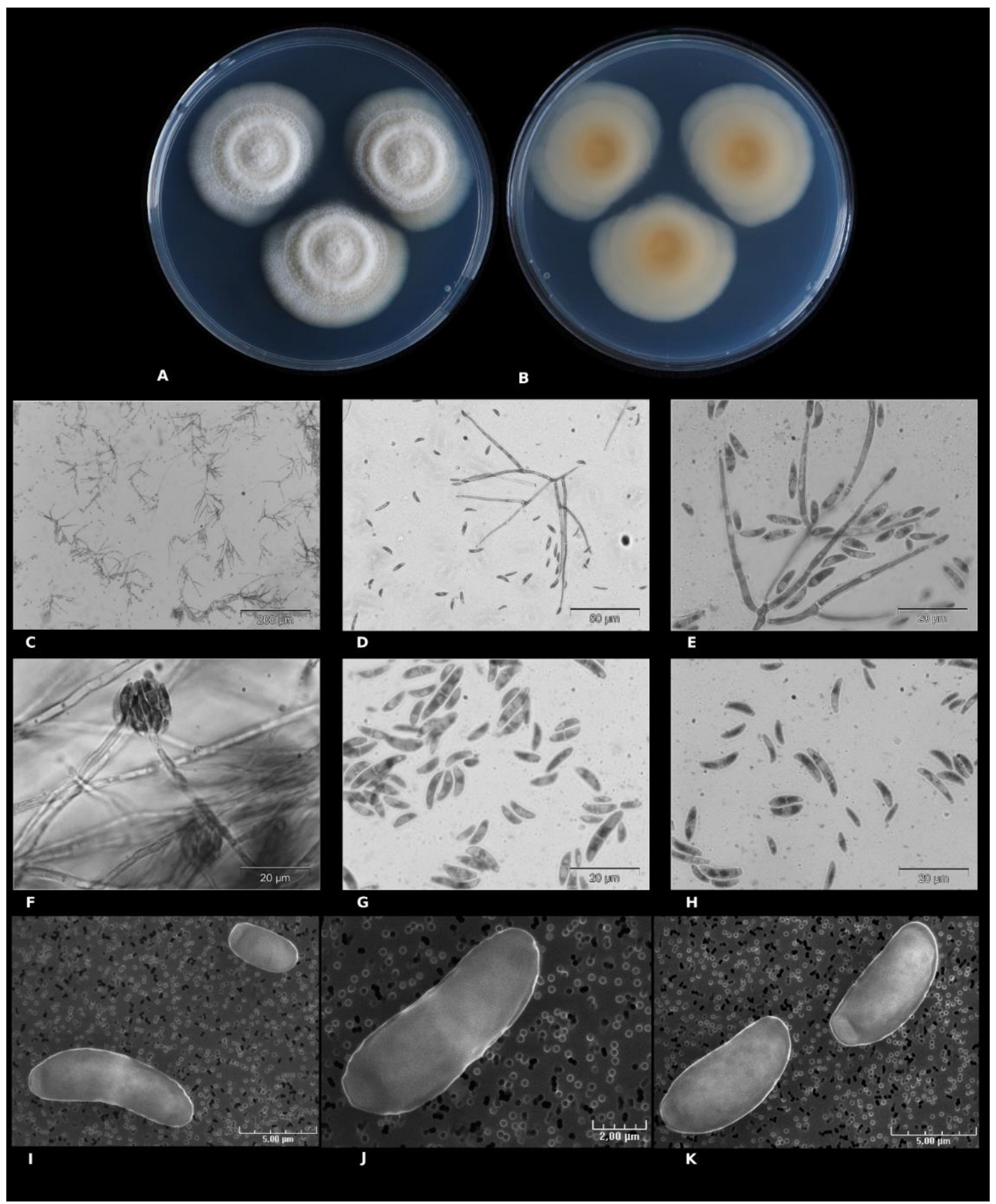

Figure 6 - Macroscopic morphological and microscopic characteristics of Longinectria verticilliforme (UBOCC-A-120043 ${ }^{\mathrm{HT}}$ ). A Colony on PDA (14 days at $\left.25^{\circ} \mathrm{C}\right)$. B Colony reverse on PDA (14 days at $\left.25^{\circ} \mathrm{C}\right)$. C-E phialides. F-K micro- and macro- conidia. $\mathrm{C}-\mathrm{H}$ microscopic acquisitions using classical microscopy, I-K: microscopic acquisitions using scanning electron microscopy.

Secondary metabolites - No known mycotoxins already described to be produced by Fusarium, Penicillium, Aspergillus or Alternaria spp. were detected. For most detected compounds, except ergosterol, extrolites could not be identified but belonged to 32 chromophore families (Table 3 ). 
These secondary metabolites included the "emon" chromophore or corresponded to extra apolar free fatty acids (including linoleic acid and probably oleic acid), mid-cyclic lipopeptides with tyrosine in the cyclic peptide as well as RED chromophore (Table 3).

Substrate and distribution - To date, the only strain isolated for this species was from an Italian cheese.

Material examined - France, isolated from an Italian cheese (Alpeggio), O. Savary, 10 Dec. 2018, holotype UBOCC-A-120043, CBS 147589.

Table 3 Production of different chromophore groups of secondary metabolites by Nectriaceae spp.

\begin{tabular}{|c|c|c|c|c|c|}
\hline Chromophore family & Identity or chromophore & $\begin{array}{l}\text { Bisifusarium } \\
\text { allantoides } \\
\text { UBOCC-A-120036 } \\
\end{array}$ & $\begin{array}{l}\text { Longinectria } \\
\text { lagenoides } \\
\text { UBOCC-A-120039 } \\
\end{array}$ & $\begin{array}{l}\text { Bisifusarium } \\
\text { penicilloides } \\
\text { UBOCC-A-120021 }\end{array}$ & $\begin{array}{l}\text { Longinectria } \\
\text { verticilliforme } \\
\text { UBOCC-A-120043 }\end{array}$ \\
\hline Alk 1-3 & indole alkaloid chromophore & 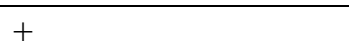 & + & + & - \\
\hline Alk 4 & indole alkaloid chromophore & - & + & - & - \\
\hline Aspham & alkylphenone chromophore & - & + & - & - \\
\hline Atrov & atrovenetin chromophore & - & + & - & - \\
\hline Cyt & tyrosine chromophore & + & + & + & + \\
\hline e1-12 & no chromophore & + & + & + & + \\
\hline e15-21 & no chromophore & + & + & + & + \\
\hline e38-48 & fatty acid & + & - & + & - \\
\hline Emon & & + & + & + & + \\
\hline Ergosterol & $\begin{array}{l}\text { (common membrane } \\
\text { constituent) }\end{array}$ & + & + & + & + \\
\hline Ffa 1-4 & fatty acid & + & + & + & + \\
\hline Flit & & - & + & - & - \\
\hline $\begin{array}{l}\text { Fluorescing compound } \\
\text { at retention time } 12.95\end{array}$ & & - & + & - & - \\
\hline Linoleic acid & linoleic acid & + & + & + & + \\
\hline Pal & & - & - & + & - \\
\hline Pyru & $\begin{array}{l}\text { pyruvoylaminobenzamide } \\
\text { chromophore }\end{array}$ & + & + & + & - \\
\hline RED & & - & - & - & + \\
\hline Snor & & + & - & + & - \\
\hline Ulm & & + & - & + & + \\
\hline Vit & & + & + & + & + \\
\hline Vor & & + & + & + & + \\
\hline $\mathbf{x x}$ & & + & - & - & + \\
\hline
\end{tabular}




\section{Discussion}

The Ascomycete Nectriaceae (Hypocreales) family includes saprotrophic and/or plant and human pathogens. Interestingly, several species within the family have been used for industrial purposes (Lombard et al. 2015). One species, namely Bisifusarium domesticum, plays an essential role for many washed-rind cheeses to reduce surface stickiness (Bachmann et al. 2003, 2005). To date, the latter species has only been isolated from cheese and was the only Nectriaceae species clearly associated with this food matrix. However, this study reports the unexpected diversity of Fusarium-like species occurring in the cheese environment, and describes four new cheeseassociated Nectriaceae species, including two that may pertain to a new genus.

While sampling washed-rind cheeses to obtain $B$. domesticum isolates on DG18 agar medium, we were firstly able to collect multiple Fusarium spp. isolates, rarely described in cheeses. In particular, isolates belonging to Fusarium babinda, F. culmorum, F. oxysporum and Fusarium solani s.l. were isolated from different cheeses although these species, considered as plant pathogens and/or soil saprophytes, are mainly recovered from soil and plant debris, but also the cause of some human infections (Leslie \& Summerell 2006, Zhang et al. 2006, Geiser et al. 2013, Summerell 2019). Obviously, the question arises about their presence on cheeses, which is likely due to undesirable environmental contaminations. Secondly, in addition to these species, several isolates with a Fusarium-like morphology were also found and further grouped into four different taxa. Based on blastn analysis using tefl sequence, they could not be assigned to Bisifusarium domesticum or to any other known species within the Nectriaceae family.

Deciding whether or not these four taxa indeed corresponded to new species, i.e. "segments of evolutionary lineages" that evolve independently (de Queiroz 1998), is not a trivial task. Different criteria exist to detect lineages that have evolved independently. However, neither the Biological Species Concept (BSC, see Anderson \& Ullrich 1979) nor the Genealogical Concordance Phylogenetic Species Recognition (Taylor et al. 2000) could be applied in this study. First, crossmating between isolates collected in this study and other strains representative of phylogenetically close species was not possible since the teleomorphs for these species are not always known. Moreover, we did not collect a sufficient number of isolates of each putative new species to consider evaluating the concordance/incongruity of multiple unlinked genes to indicate a lack of genetic exchanges between lineages evolving independently as proposed by the GC-PSR criterium (Taylor et al. 2000) or even to observe the number of shared/unique single nucleotide polymorphisms (SNPs) among/within lineages. Therefore, this study focused on the use of multiple locus phylogenetic reconstructions that could identify independent clades and inform about the nucleotide divergences between taxa. By using the same set of loci as the one used by Lombard et al. (2015), that was publicly available at the beginning of this study, we were able to assess the phylogenetic placement of the four taxa representing putative species within the Nectriaceae family, and evaluate the nucleotide divergence between these taxa and the other phylogenetically close species and genera.

It is noteworthy that the circumscription of the Fusarium genus has recently been highly debated (Crous et al. 2021 vs. Geiser et al. 2021), mainly with two concepts: (i) a broad Fusarium concept (O'Donnell et al. 2020, Geiser et al. 2021) corresponding to a monophyletic group initiated at node F1 (node F1 in Geiser et al. 2013). This hypothesis was recently supported by a 19-gene phylogeny (Geiser et al. 2021) which showed a better phylogenetic support for node F1 than for other internal nodes. In addition, different practical arguments provided in O'Donnell et al. 2020 encourage the scientific community to use the broad Fusarium concept. (ii) A second concept with a Fusarium genus corresponding to a monophyletic group initiated at node F3 (node F3 in Geiser et al. 2013) that excludes the following genera: Albonectria, Cyanonectria, Geejayessia, Neocosmospora, Rectifusarium and Bisifusarium. The latter proposition brings forward the strongly supported monophyly of these different genera as well as its morphological, biochemical and ecological consistency that contrasts with the absence of synapomorphy when considering the broad Fusarium concept.

Pending adoption of a consensus taxonomy, we named taxa based on the nomenclature used by Lombard et al. (2015), although future studies may lead to genus name changes. This approach 
also led to the proposal of a new genus (i.e. Longinectria) within the Nectriaceae family based on the definition of Crous et al. (2021), O’Donnell et al. (2020), Geiser et al. (2021).

In the context of this study, after a preliminary identification based on tefl sequencing, a phylogenetic analysis was performed comparing the ten-locus sequence set used by Lombard et al. (2015), namely acl1, act, cmdA, his3, ITS, LSU, rpb1, rpb2, tef1 and tub2, from thirteen isolates, eleven corresponding to unidentified isolates with a Fusarium-like morphology and the two others where $F$. babinda and $F$. solani s.l. isolates. Finally, B. biseptatum CBS $110311^{\mathrm{T}}$, missing from Lombard et al. 2015 analysis, and B. dimerum MNHN-RF-05625 , as an internal control, were also analysed. The obtained sequences were compared with the corresponding sequences from 57 strains issued from the dataset analyzed by the latter authors and supplemented with $B$. tonghuanum CGMCC $3.17369^{\mathrm{T}}$ (Sun et al. 2017) sequences (6/10 loci available). A complementary analysis was then performed with the sequences of six loci, namely ITS, LSU, rpb1, rpb2, tef1 and tub2, from the ten isolates identified as pertaining to the Bisifusarium genus. This was done to ensure that the missing sequence data for the recently described B. tonghuanum (Sun et al. 2017) had no impact on the positioning of some of our isolates as new Bisifusarium species.

Firstly, the ten loci analysis showed that six of the Fusarium-like cheese isolates clustered into two groups, the first one (i) containing UBOCC-A-120021, UBOCC-A-120034 and VTT-D-042022 strains, and the second one (ii) UBOCC-A-120035, UBOCC-A-120036 and UBOCC-A-120037 strains. These groups positioned within the supported Bisifusarium genus (Lombard et al. 2015) and were phylogenetically separated from other already described species within this genus as well as other Fusarium species. Moreover, the complementary analysis focused on the Bisifusarium genus (using six loci) confirmed the same topology. These two species formed a sister clade of $B$. tonghuanum. The nucleotide differences between these isolates and $B$. tonghuanum CGMCC $3.17369^{\mathrm{T}}$ (299/5134 (90.2\% identity) and 325/5134 (89.2\% identity) nucleotides for B. penicilloides and B. allantoides, respectively), as well as with the other Bisifusarium species (e.g. 566/5134 (88.5\% identity) and 571/5134 (88.4\% identity) nucleotide differences with B. biseptatum CBS $110311^{\mathrm{T}}$ ), supports the existence of two new Bisifusarium species. Indeed, among Bisifusarium, several species have nucleotide differences that are 5-fold lower than between $B$. allantoides and $B$. penicilloides (e.g. 73/5134 nucleotides (98.5\% identity) between B. penzigii CBS $317.34^{\mathrm{T}}$ and B. biseptatum CBS $110311^{\mathrm{T}}$ ). Following morphological observations, the names Bisifusarium allantoides and $B$. penicilloides were proposed for these two new species. These two species were isolated from cheese rinds and their growth was confirmed at temperatures as low as $5^{\circ} \mathrm{C}$ thus compatible with cheesemaking conditions. Interestingly, among B. penicilloides isolates, one strain was also isolated from the environment (sandstone building). This presumptive identification proximity based on macromorphological observation was confirmed using the ten loci sequencing phylogenetic analysis. This strain, isolated from the outer surface of a sandstone building, could provide key information about the environmental origin of this species. Indeed, this association to sandstone might suggest that this species is naturally found in some cheese, via its presence in ripening cellars (i.e. on walls or building surfaces). It is noteworthy that the genus Bisifusarium might actually include a larger number of species than the ten Bisifusarium spp. presented in this study. Indeed, among the five strains assigned to unnamed Bisifusarium species in Sun et al. (2017), a preliminary Maximum Likelihood analysis was carried out on a reduced dataset comprising the only sequences available for four loci for these strains (ITS, LSU, tef1 and tub2). The results suggested that these strains might pertain to new Bisifusarium species (data not shown).

As for the other isolates, the position of the genetic cluster (iii) consisting of UBOCC-A120038, UBOCC-A-120039, UBOCC-A-120041 and ESE-00140, on the one hand, and (iv) UBOCC-A-120043, on the other hand, that formed two branches within an independent sister clade of a clade grouping Albonectria, Fusarium, Geejayessia, Cyanonectria and Neocosmospora genera (as described by Lombard et al. 2015), is compatible with their affiliation to two new species within a new genus. The minimal nucleotide divergence (566/5134 nucleotides) existing between this clade and its sister clade, grouping Albonectria, Fusarium, Geejayessia, Cyanonectria and Neocosmospora genera, is similar or higher than those existing between genera of the latter clade (e.g. minimal 
nucleotide divergence 554/5134 nucleotide between Cyanonectria and Neocosmospora). Furthermore, nucleotide differences (483/5134 nucleotides, 90.2\% identity) occurring between UBOCC-A-120043 and UBOCC-A-120038 / UBOCC-A-120039 / UBOCC-A-120041 / ESE-00140 supported the fact that the two branches actually correspond to two different species within the genus. According to morphological characters of these five isolates, namely the observed length of the phialides, which appear to be longer than phialides commonly observed within the Nectriaceae, the name Longinectria (gen. nov.) is proposed in the present study for members of this genus. Then, following morphological observations, the names Longinectria verticilliforme and L. lagenoides were proposed for these two new species. Noteworthy, Crous et al. (2021) very recently described several new Nectriaceae genera, among which two (Luteonectria gen. nov. and Nothofusarium gen. nov.) are positioned close to the previously cited genus. However, the phylogenetic divergence between the Longinectria spp. (based on a Maximum Likelihood analysis using the accessible sequence, Supplementary Fig. 2) and these new genera, as well as morphological differences regarding phialides and conidia, agreed with the existence of another new genus (i.e. Longinectria gen. nov.) within the Nectriaceae family. Otherwise, temperature impact was investigated on these two species and, interestingly, growth was observed at $5^{\circ} \mathrm{C}$ while no growth occured at $30^{\circ} \mathrm{C}$ and $37^{\circ} \mathrm{C}$ on PDA. These observations are clearly in agreement with cheese production, as reported for B. domesticum for which a maximum growth temperature $\left(\mathrm{T}_{\max }\right)$ was estimated to be $30^{\circ} \mathrm{C}$ (Schroers et al. 2009).

Overall, the present study clearly highlighted the unexpected diversity of Nectriaceae in cheeses which, up to now, were considered to be solely colonized by B. domesticum that is used as a technological auxiliary for its "anticollanti" ability (Bachmann et al. 2003, 2005). As shown in this study, one can note that even microbial ecosystems that have been associated with human activity for centuries, and quite extensively studied, are still sources to discover novel microbial diversity. The obtained data clearly suggests that cheeses, which is obviously the case for other fermented foods, might harbour much higher diversity than expected. By sampling a relatively small panel of washed-rind cheeses $(n=56)$, we identified four new species within the Nectriaceae family, a family that is best documented for pathogenic species (plant and/or human) (Lombard et al. 2015). This study is therefore a hint that, besides the most emblematic Penicillium species, there is still much to discover about cheese microbiota and more specifically the fungal communities of fermented foods. Regarding the four species described in the present study, it should be noted that the obtained results on extrolite production tend, so far, towards a safety status (in the sense of the European Qualified Presumption of Safety -QPS- or the US Generally Recognized As Safe -GRAS- statuses) of these isolates as no known mycotoxins, metabolites largely produced by Fusarium genus members (e.g. F . graminearum or $F$. avenaceum), were observed in the tested conditions. However, as some metabolites are unknown, this aspect should be further investigated. In the future, it could also be of interest to assess the respective functional diversity of these species to better understand their metabolic potential and to use comparative genomics to potentially identify cheese adaptation footprints and elucidate their role in cheese.

\section{Acknowledgments}

We thank all people who kindly sent us the various cheeses analyzed in this study (Pr. Luca Cocolin, Pr. Jérôme Mounier, Dr. Guillaume Dubrulle, MR Sten Marc) as well as those who have provided isolates from their research studies (Dr. Miguel Alvarez, Dr. Baltasar Mayo and Franck Deniel). Otherwise, we would like to thank Mr. Philippe Elies of the PIMMS platform at the Université de Bretagne Occidentale for electronic microscopy acquisitions and Maud Etienne Duplessis for her help regarding the new species names. We would also like to thank Erwan Corre for his help for developing bioinformatics workflows (ABiMS Platform, Station Biologique de Roscoff). Finally, we are also grateful to Pr. Pedro W. Crous, Dr. Lorenzo Lombard and Dr. Marcelo Sandoval-Denis for helpful discussions and for providing the sequences of recently described new Nectriaceae genera. 


\section{Funding}

OS benefited from a Ph. D grant from the Région Bretagne and the French Ministère de l'Enseignement Supérieur, de la Recherche et de l'Innovation. This work was co-funded by the French Dairy Interbranch Organization (CNIEL) (DIFUS Project, 2019_00627).

\section{Declarations}

\section{Conflicts of interest/Competing interests}

The authors declare that they have no known competing financial interests or personal relationships that could have appeared to influence the work reported in this paper.

\section{Availability of data and material}

The sequences generated and used in this phylogenetic analysis were deposited in GenBank under accession numbers detailed in this paper and the multi-gene phylogenetic analyses were deposited in TreeBASE. The holotype strains were made available at the UBOCC (Université de Bretagne Occidentale, France) and CBS-KNAW (Westerdijk Fungal Biodiversity Institute, The Netherland) culture collections.

\section{Authors' contributions}

EC, MC, and JLJ obtained the funding, supervised the study and designed the experiments. OS performed the experimental work and analysed all data. OS and JLJ performed phylogenetics analysis. OS and MC were involved in extrolite preparations while JCF performed all extrolite analyses on fungal cultures. JR performed some of the Fusarium-like fungi isolations and molecular identifications. PN supervised OS for microscopical observations and JLJ contributed to the new species morphological descriptions. OS drafted the manuscript and all co-authors edited and proofread the manuscript.

\section{References}

Anderson JB, Ullrich RC. 1979 - Biological species of Armillaria mellea in North America. Mycologia 71: 402-414.

Bachmann H-P, Bobst C, Bütikofer U, Casey MG et al. 2005 - Occurrence and significance of Fusarium domesticum alias Anticollanti on smear-ripened cheeses. Lebensmittel-Wissenschaft und-Technologie 38: 399-407.

Bachmann H-P, Bobst C, Bütikofer U, Dalla M et al. 2003 - Sticky cheese smear and natural white mould. Milchwissenschaft 58: 117-232

Bodinaku I, Shaffer J, Connors A, Steenwyk J et al. 2019 - Rapid phenotypic and metabolomic domestication of wild Penicillium molds on cheese. In: MBio 10: e02445-19.

Cheeseman K, Ropars J, Renault P, Dupont J et al. 2014 - Multiple recent horizontal transfers of a large genomic region in cheese making fungi. Nat Commun 5:2876.

Crous PW, Lombard L, Sandoval-Denis M, Seifert KA et al. 2021 - Fusarium: more than a node or a foot-shaped basal cell. Studies in Mycology 98:100116.

de Queiroz K. 1998 - The general lineage concept of species, species criteria, and the process of speciation. In: Howard DJ, Berlocher SH (eds) Endless Forms: Species and Speciation. Oxford University Press, pp. 57-75

Deng W, Maust BS, Nickle DC et al. 2010 - DIVEIN: a web server to analyze phylogenies, sequence divergence, diversity, and informative sites. Biotechniques 48: 405-408.

Dugat-Bony E, Garnier L, Denonfoux J, Ferreira S et al. 2016 - Highlighting the microbial diversity of 12 French cheese varieties. International Journal of Food Microbiology 238: 265-273.

Dumas E, Feurtey A, Rodríguez de la Vega RC, Le Prieur S et al. 2020 - Independent domestication events in the blue-cheese fungus Penicillium roqueforti. Mol Ecol. 29:2639-2660. 
Dupont J, Dequin S, Giraud T, Le Tacon F et al. 2017 - Fungi as a Source of Food. Microbiology Spectrum 5:FUNK-0030-2016.

Fox PF, Guinee TP. 2020 - Cheese: Overview. In: Reference module in food science. Elsevier.

Frisvad JC, Thrane U. 1987 - Standardized high-performance liquid chromatography of 182 mycotoxins and other fungal metabolites based on alkylphenone retention indices and UV-VIS spectra (diode array detection). J Chromatogr 404: 195-214.

Frisvad JC, Thrane U. 1993 - Chapter 8 Liquid column chromatography of mycotoxins. In: Betina V (ed) Journal of Chromatography Library. Elsevier, pp. 253-372

Geiser DM, Al-Hatmi A, Aoki T, Arie T et al. 2021 - Phylogenomic analysis of a $55.1 \mathrm{~kb}$ 19-gene dataset resolves a monophyletic Fusarium that includes the Fusarium solani Species Complex. Phytopathology.

Geiser DM, Aoki T, Bacon CW, Baker S et al. 2013 - One fungus, one name: defining the genus Fusarium in a scientifically robust way that preserves long standing use. Phytopathology 103: 400-408.

Gerlach W, Nirenberg H. 1982 - The genus Fusarium - a pictorial atlas. Mitteilungen aus der Biologischen Bundesanstalt fur Land- und Forstwirtschaft Berlin-Dahlem 209: 1-406.

Gillot G, Jany J-L, Poirier E, Maillard MB et al. 2017 - Functional diversity within the Penicillium roqueforti species. Int J Food Microbiol 241: 141-150.

Hermet A, Méheust D, Mounier J, Barbier G et al. 2012 - Molecular systematics in the genus Mucor with special regards to species encountered in cheese. Fungal Biology 116: 692-705.

Hermet A, Mounier J, Keravec M, Vasseur V et al. 2014 - Application of capillary electrophoresis single-stranded conformation polymorphism (CE-SSCP) analysis for identification of fungal communities in cheese. Food Microbiology 41: 82-90.

Irlinger F, Layec S, Hélinck S, Dugat-Bony E. 2015 - Cheese rind microbial communities: diversity, composition and origin. FEMS Microbiol Lett 362: 1-11.

Katoh K, Rozewicki J, Yamada KD. 2019 - MAFFT online service: multiple sequence alignment, interactive sequence choice and visualization. Briefings in Bioinformatics 20: 1160-1166.

Kildgaard S, Mansson M, Dosen I, Klitgaard A et al. 2014 - Accurate dereplication of bioactive secondary metabolites from marine-derived fungi by UHPLC-DAD-QTOFMS and a MS/HRMS library. Mar Drugs 12: 3681-3705.

Klitgaard A, Iversen A, Andersen MR, Larsen TO et al. 2014 - Aggressive dereplication using UHPLC-DAD-QTOF: screening extracts for up to 3000 fungal secondary metabolites. Anal Bioanal Chem 406: 1933-1943.

Kuraku S, Zmasek CM, Nishimura O, Katoh K. 2013 - aLeaves facilitates on-demand exploration of metazoan gene family trees on MAFFT sequence alignment server with enhanced interactivity. Nucleic Acids Research 41: W22-W28.

Lebreton A, Corre E, Jany J-L, Brillet-Guéguen L et al. 2020 - Comparative genomics applied to Mucor species with different lifestyles. BMC Genomics 21: 235.

Leslie JF, Summerell BA. 2006 - The Fusarium Laboratory Manual, Blackwell Publishing.

Lombard L, van der Merwe NA, Groenewald JZ, Crous PW. 2015 - Generic concepts in Nectriaceae. Studies in Mycology 80: 189-245.

Morin-Sardin S, Rigalma K, Coroller L, Jany J-L et al. 2016 - Effect of temperature, pH, and water activity on Mucor spp. growth on synthetic medium, cheese analog and cheese. Food Microbiol 56: 69-79.

Nielsen KF, Månsson M, Rank C, Frisvad J et al. 2011 - Dereplication of microbial natural products by LC-DAD-TOFMS. J Nat Prod 74: 2338-2348.

O’Donnell K, Al-Hatmi AMS, Aoki T, Brankovics B et al. 2020 - No to Neocosmospora: phylogenomic and practical reasons for continued inclusion of the Fusarium solani Species Complex in the genus Fusarium. mSphere 5: e00810-20.

Rambaut A. 2006 - FigTree. http://tree.bio.ed.ac.uk/software/figtree/ (Accessed on April 19, 2021).

Rambaut A, Drummond AJ, Xie D, Baele G et al. 2018 - Posterior summarization in Bayesian phylogenetics using Tracer 1.7. Syst Biol 67: 901-904. 
Ropars J, Cruaud C, Lacoste S, Dupont J. 2012 - A taxonomic and ecological overview of cheese fungi. Int J Food Microbiol 155: 199-210.

Ropars J, Didiot E, Vega RCR de la, Bennetot B et al. 2020 - Domestication of the emblematic white cheese-making fungus Penicillium camemberti and its diversification into two varieties. bioRxiv 2020.02.12.945238.

Ropars J, Lo Y-C, Dumas E, Snirc A et al. 2016 - Fertility depression among cheese-making Penicillium roqueforti strains suggests degeneration during domestication. Evolution 70: 2099-2109.

Ropars J, López-Villavicencio M, Snirc A, Lacoste S et al. 2017 - Blue cheese-making has shaped the population genetic structure of the mould Penicillium roqueforti. PLoS One 12: e0171387.

Ropars J, Rodríguez de la Vega RC, López-Villavicencio M, Gouzy J et al. 2015 - Adaptive horizontal gene transfers between multiple cheese-associated fungi. Curr Biol 25: 2562-2569.

Sandoval-Denis M, Guarnaccia V, Polizzi G, Crous PW. 2018 - Symptomatic Citrus trees reveal a new pathogenic lineage in Fusarium and two new Neocosmospora species. Persoonia 40: 125.

Sandoval-Denis M, Lombard L, Crous PW. 2019 - Back to the roots: a reappraisal of Neocosmospora. Persoonia 43: 90-185.

Schroers H-J, O’Donnell K, Lamprecht SC, Kammeyer PL et al. 2009 - Taxonomy and phylogeny of the Fusarium dimerum species group. Mycologia 101: 44-70.

Stamatakis A. 2014 - RAxML version 8: a tool for phylogenetic analysis and post-analysis of large phylogenies. Bioinformatics 30: 1312-1313.

Stamatakis A. 2016 - The RAxML v8. 2. X Manual. Heidelberg Institute for Theoretical Studies.

Suihko M-L, Alakomi H-L, Gorbushina A, Fortune I et al. 2007 - Characterization of aerobic bacterial and fungal microbiota on surfaces of historic Scottish monuments. Syst Appl Microbiol 30: 494-508.

Summerell BA. 2019 - Resolving Fusarium: Current Status of the Genus. Annu Rev Phytopathol 57: 323-339.

Sun B-D, Zhou Y-G, Chen A. 2017 - Bisifusarium tonghuanum (Nectriaceae), a novel species of Fusarium-like fungi from two desert oasis plants. Phytotaxa 317: 123.

Swofford DL. 2003 - Paup*. Phylogenetic analysis using parsimony and other methods. Version 4. Sinauer Associates, Sunderland, Massachusetts.

Taylor JW, Jacobson DJ, Kroken S, Kasuga T et al. 2000 - Phylogenetic species recognition and species concepts in fungi. Fungal Genetics and Biology 31: 21-32.

Wolfe BE, Button JE, Santarelli M, Dutton RJ. 2014 - Cheese rind communities provide tractable systems for in situ and in vitro studies of microbial diversity. Cell 158: 422-433.

Yang Z. 2006 - Computational Molecular Evolution. Oxford: Oxford University Press.

Zhang N, O'Donnell K, Sutton DA, Nalim FA et al. 2006 - Members of the Fusarium solani species complex that cause infections in both humans and plants are common in the environment. $\mathrm{J}$ Clin Microbiol 44: 2186-2190.

Zwietering MH, Jongenburger I, Rombouts FM, van 't Riet K. 1990 - Modeling of the bacterial growth curve. Appl Environ Microbiol 56: 1875-1881 